\title{
Global Dynamics of Secondary DENV Infection with Diffusion
}

\author{
A. M. Elaiw $\mathbb{D}^{1,2}$ and A. S. Alofi ${ }^{1}$ \\ ${ }^{1}$ Department of Mathematics, Faculty of Science, King Abdulaziz University, P.O. Box 80203, Jeddah 21589, Saudi Arabia \\ ${ }^{2}$ Department of Mathematics, Faculty of Science, Al-Azhar University, Assiut Branch, Assiut, Egypt
}

Correspondence should be addressed to A. M. Elaiw; a_m_elaiw@yahoo.com

Received 28 January 2021; Accepted 18 June 2021; Published 28 June 2021

Academic Editor: Nan-Jing Huang

Copyright (C) 2021 A. M. Elaiw and A. S. Alofi. This is an open access article distributed under the Creative Commons Attribution License, which permits unrestricted use, distribution, and reproduction in any medium, provided the original work is properly cited.

\begin{abstract}
During the past eras, many mathematicians have paid their attentions to model the dynamics of dengue virus (DENV) infection but without taking into account the mobility of the cells and DENV particles. In this study, we develop and investigate a partial differential equations (PDEs) model that describes the dynamics of secondary DENV infection taking into account the spatial mobility of DENV particles and cells. The model includes five nonlinear PDEs describing the interaction among the target cells, DENV-infected cells, DENV particles, heterologous antibodies, and homologous antibodies. In the beginning, the well-posedness of solutions, including the existence of global solutions and the boundedness, is justified. We derive three threshold parameters which govern the existence and stability of the four equilibria of the model. We study the global stability of all equilibria based on the construction of suitable Lyapunov functions and usage of Lyapunov-LaSalle's invariance principle (LLIP). Last, numerical simulations are carried out in order to verify the validity of our theoretical results.
\end{abstract}

\section{Introduction}

Mathematical models and their analysis have been proven to be an efficient and significant approach to understand the within-host dynamics of viral infections such as dengue virus (DENV), human immunodeficiency virus (HIV), hepatitis $\mathrm{C}$ and $\mathrm{B}$ virus ( $\mathrm{HCV} / \mathrm{HBV})$, human $\mathrm{T}$ lymphotropic virus type I (HTLV-I), and recently, severe acute respiratory syndrome coronavirus 2 (SARS-CoV-2). $\mathrm{DENV}$ is the casual of dengue fever which is one of the morbidity and mortality diseases. It can transmit to humans via Aedes aegypti and Aedes albopictus mosquitoes. Annually, about 50-100 million infected individuals by DENV are reported worldwide. The most epidemic regions are sub-Saharan Africa and Southeast Asia [1]. Several symptoms of dengue that can appear on the infected individual are high fever, vomiting, nausea, joint pains, headache, and pain behind the eyes [2]. DENV aims and infects the following types of cells: monocytes, dendritic cells, hepatocytes, macrophages, and mast cells
[3-6]. There are four serologically various dengue viruses DENV (1-4) that can infect the human [7]. When a DENV enters the human body first time, the immune response is enhanced [8]. Cytotoxic T lymphocytes (CTLs) and antibody immune responses are two main components of the immune system against viruses. CTLs destroy the DENV-infected cells, while antibodies kill DENV particles and clear it from the body.

During the recent years, several mathematical models have been developed which describe within-host DENV primary infection [9-17]. These models are based on the virus dynamics model introduced by Nowak and Bangham [18]. The World Health Organization (WHO) [19] has reported that an infected individual by one serotype will have lifelong immunity against that serotype but only temporary and partial cross-immunity to the other three serotypes. Mathematical models of DENV dynamics pertaining to secondary infection with another serotype have been developed in [20-25]. Gujarati and Ambika [20] have formulated the following DENV infection model: 


$$
\begin{aligned}
\frac{\mathrm{d} K(t)}{\mathrm{d} t} & =\delta-\mu K(t) M(t)-\xi K(t), \\
\frac{\mathrm{d} L(t)}{\mathrm{d} t} & =\mu K(t) M(t)-\varrho L(t), \\
\frac{\mathrm{d} M(t)}{\mathrm{d} t} & =\tau L(t)-\eta M(t)-\varpi_{1} M(t) N(t)-\omega_{2} M(t) P(t), \\
\frac{\mathrm{d} B(t)}{\mathrm{d} t} & =\beta-\gamma B(t)+\varphi B(t) M(t), \\
\frac{\mathrm{d} N(t)}{\mathrm{d} t} & =\vartheta \epsilon B(t)-\varkappa_{1} N(t) M(t)-\alpha_{1} N(t), \\
\frac{\mathrm{d} P(t)}{\mathrm{d} t} & =\epsilon B(t)-\varkappa_{2} P(t) M(t)-\alpha_{2} P(t),
\end{aligned}
$$

where $t>0$ is the time, and $K(t), L(t), M(t), B(t), N(t)$, and $P(t)$ are the concentrations of the target cells, DENV-infected cells, DENV particles, B cells, heterologous antibody previously formed on primary infection, and homologous antibody against the new virus serotype of the secondary infection, respectively. The parameter $\delta$ represents the creation rate of the target cells. The DENV particles infect the target cells at rate $\mu K M$. The DENV-infected cells produce viruses at rate $\tau L$. The B cells are created at constant rate $\beta$ and proliferated at rate $\varphi B M$. The death rates of the compartments $K, L, M, B, N$, and $P$ are given by $\xi K, \varrho L, \eta M$, $\gamma B, \alpha_{1} N$, and $\alpha_{2} P$, respectively. The two types of antibodies $N$ and $P$ are generated from the B cells at rates $\vartheta \epsilon B$ and $\epsilon B$ and neutralize the DENV at rates $\omega_{1} M N$ and $\omega_{2} M P$. The terms $\varkappa_{1} N M$ and $\varkappa_{1} P M$ represent the rates at which antibody virus complex affects the antibody growth. $\vartheta \in(0,1)$ is a correlation factor that quantifies the similarity between the individual serotypes. We observe that the global stability of the models presented in [20-25] is not well studied.

All of the DENV infection models in the abovementioned works are given by ordinary or delay differential equations under the assumption that the cells and DENV particles are well mixed. Spatial structure plays an important role in understanding the dynamical behavior of viral infection within a host. In recent years, spatial dependence has been incorporated into mathematical models of several viral infections such as hepatitis B virus (HBV) [26], hepatitis C virus (HCV) $[27,28]$, human immunodeficiency virus (HIV) [29-31], and human T lymphotropic virus type I (HTLV-I) [32]. To the best of our knowledge, the DENV infection model with diffusion has not been studied before. Therefore, the aim of the present study is to focus on the dynamical behavior of DENV infection with diffusion. Following the work of Hattaf [33], our proposed model takes into account the spatial mobility of all compartments.

\section{Mathematical DENV Dynamics Model}

We develop a DENV infection model with secondary infection and diffusion as

$$
\begin{aligned}
\frac{\partial K(u, t)}{\partial t}= & d_{K} \Delta K(u, t)+\delta-\mu K(u, t) M(u, t)-\xi K(u, t), \\
\frac{\partial L(u, t)}{\partial t}= & d_{L} \Delta L(u, t)+\mu K(u, t) M(u, t)-\varrho L(u, t), \\
\frac{\partial M(u, t)}{\partial t}= & d_{M} \Delta M(u, t)+\tau L(u, t)-\eta M(u, t) \\
& -\Phi_{1} M(u, t) N(u, t)-\Phi_{2} M(u, t) P(u, t), \\
\frac{\partial N(u, t)}{\partial t}= & d_{N} \Delta N(u, t)+\lambda_{1} M(u, t) N(u, t)-\alpha_{1} N(u, t), \\
\frac{\partial P(u, t)}{\partial t}= & d_{P} \Delta P(u, t)+\lambda_{2} M(u, t) P(u, t)-\alpha_{2} P(u, t),
\end{aligned}
$$

where $u \in \Gamma$ is the position. The heterologous and homologous antibodies are activated at rates $\lambda_{1} M N$ and $\lambda_{2} M P$, respectively. Here, $\Delta$ is the Laplacian operator and $d_{x}$ is the diffusion coefficient, where $x \in\{K, L, M, N, P\}$. The spatial domain $\Gamma \subset \mathbb{R}^{m}$ (where $m \geq 1$ ) is bounded and connected; moreover, its boundary $\partial \Gamma$ is smooth.

The initial conditions are given by

$$
\begin{aligned}
K(u, 0) & =\mathscr{G}_{1}(u), \\
L(u, 0) & =\mathscr{G}_{2}(u), \\
M(u, 0) & =\mathscr{G}_{3}(u), \\
N(u, 0) & =\mathscr{G}_{4}(u), \\
P(u, 0) & =\mathscr{G}_{5}(u), \quad u \in \bar{\Gamma},
\end{aligned}
$$

where $\mathscr{G}_{\ell}(u) \geq 0, \ell=1, \ldots, 5$, are the continuous functions. In addition, we take the following homogeneous Neumann boundary conditions:

$$
\frac{\partial K}{\partial \overrightarrow{\mathscr{B}}}=\frac{\partial L}{\partial \overrightarrow{\mathscr{B}}}=\frac{\partial M}{\partial \overrightarrow{\mathscr{B}}}=\frac{\partial N}{\partial \overrightarrow{\mathscr{B}}}=\frac{\partial P}{\partial \overrightarrow{\mathscr{B}}}=0, \quad t>0, u \in \partial \Gamma,
$$

where $\partial / \partial \overrightarrow{\mathscr{B}}$ is the outward normal derivative on the boundary $\partial \Gamma$. These boundary conditions indicate that cells and viruses cannot cross the isolated boundary [34].

\section{Well-Posedness of Solutions}

Theorem 1. Assume that $d_{K}=d_{L}=d_{M}=d_{N}=d_{P}=\tilde{d}$. Then, models (2)-(6) with any initial satisfying (7) has a unique, nonnegative, and bounded solution defined on $\bar{\Gamma} \times[0,+\infty)$.

Proof. We denote $\mathscr{X}=B U C\left(\bar{\Gamma}, \mathbb{R}^{5}\right)$, the set of all bounded and uniformly continuous functions from $\bar{\Gamma}$ to $\mathbb{R}^{5}$, with norm $\|\theta\|_{\mathscr{X}}=\sup _{u \in \bar{\Gamma}}|\theta(u)|$. We define the positive cone $\mathscr{X}_{+}=B U C\left(\bar{\Gamma}, \mathbb{R}_{+}^{5}\right) \subset \mathscr{X}$ which induces a partial order on $\mathscr{X}$. This shows that the space $\left(\mathscr{X},\|\cdot\|_{\mathscr{X}}\right)$ is a Banach lattice $[35,36]$. 
For any initial data $\mathscr{G}=\left(\mathscr{G}_{1}, \mathscr{G}_{2}, \mathscr{G}_{3}, \mathscr{G}_{4}, \mathscr{G}_{5}\right)^{T} \in \mathscr{X}_{+}$, we define $H=\left(H_{1}, H_{2}, H_{3}, H_{4}, H_{5}\right)^{T}: \mathscr{X}_{+} \longrightarrow \mathscr{X}$ by

$$
\begin{aligned}
& H_{1}(\mathscr{G})(u)=\delta-\mu \mathscr{G}_{1}(u) \mathscr{G}_{3}(u)-\xi \mathscr{G}_{1}(u) \\
& H_{2}(\mathscr{G})(u)=\mu \mathscr{G}_{1}(u) \mathscr{G}_{3}(u)-\varrho \mathscr{G}_{2}(u) \\
& H_{3}(\mathscr{G})(u)=\tau \mathscr{G}_{2}(u)-\eta \mathscr{G}_{3}(u)-\omega_{1} \mathscr{G}_{3}(u) \mathscr{G}_{4}(u)-\omega_{2} \mathscr{G}_{3}(u) \mathscr{G}_{5}(u) \\
& H_{4}(\mathscr{G})(u)=\lambda_{1} \mathscr{G}_{3}(u) \mathscr{G}_{4}(u)-\alpha_{1} \mathscr{G}_{4}(u) \\
& H_{5}(\mathscr{G})(u)=\mathscr{G}_{3}(u) \mathscr{G}_{5}(u)-\alpha_{2} \mathscr{G}_{5}(u)
\end{aligned}
$$

It is clear that $H$ is locally Lipschitz on $\mathscr{X}_{+}$. We can rewrite systems (2)-(6) with initial conditions (7) and boundary conditions (8) as the following abstract functional differential equation:

$$
\left\{\begin{array}{l}
\frac{\mathrm{d} \overline{\mathscr{U}}}{\mathrm{d} t}=\Theta \overline{\mathscr{U}}+H(\overline{\mathscr{U}}), \quad t>0, \\
\overline{\mathscr{U}}(0)=\mathscr{G} \in \mathscr{X}_{+},
\end{array}\right.
$$

where $\overline{\mathscr{U}}=(K, L, M, N, P)^{T}$ and $\Theta \overline{\mathcal{U}}=\left(d_{K} \Delta K, d_{L} \Delta L, d_{M}\right.$ $\left.\Delta M, d_{N} \Delta N, d_{P} \Delta P\right)^{T}$. One can show that

$$
\lim _{q \longrightarrow 0^{+}} \frac{1}{q} \operatorname{dist}\left(\mathscr{G}(0)+q H(\mathscr{G}), \mathscr{X}_{+}\right)=0, \quad \forall \mathscr{G} \in \mathscr{X}_{+} .
$$

Hence, for any $\mathscr{G} \in \mathscr{X}_{+}$, systems (2)-(6) with (7)-(8) has a unique nonnegative mild solution $(K(u, t), L(u, t)$, $M(u, t), N(u, t), P(u, t))$ defined on $\bar{\Gamma} \times\left[0, \mathscr{T}_{m}\right)$, where $\left[0, \mathscr{T}_{m}\right)$ is the maximal existence time interval on which the solution exists [35-37]. In addition, this solution also is a classical solution for the given problem.

We define

$$
\begin{aligned}
\Psi(u, t)= & K(u, t)+L(u, t)+\frac{\varrho}{2 \tau} M(u, t)+\frac{\varrho \Phi_{1}}{2 \tau \lambda_{1}} N(u, t) \\
& +\frac{\varrho \Phi_{2}}{2 \tau \lambda_{2}} P(u, t) .
\end{aligned}
$$

Then, using systems (2)-(6), we obtain

$$
\begin{aligned}
\frac{\partial \Psi(u, t)}{\partial t}= & d_{K} \Delta K(u, t)+\delta-\mu K(u, t) M(u, t)-\xi K(u, t) \\
& +d_{L} \Delta L(u, t)+\mu K(u, t) M(u, t)-\varrho L(u, t), \\
& \frac{\varrho}{2 \tau}\left[d_{M} \Delta M(u, t)+\tau L(u, t)-\eta M(u, t)-\varpi_{1} M(u, t) N(u, t)-\varpi_{2} M(u, t) P(u, t)\right] \\
& +\frac{\varrho \Phi_{1}}{2 \tau \lambda_{1}}\left[d_{N} \Delta N(u, t)+\lambda_{1} M(u, t) N(u, t)-\alpha_{1} N(u, t)\right] \\
& +\frac{\varrho \Phi_{2}}{2 \tau \lambda_{2}}\left[d_{P} \Delta P(u, t)+\lambda_{2} M(u, t) P(u, t)-\alpha_{2} P(u, t)\right] .
\end{aligned}
$$

Since $d_{K}=d_{L}=d_{M}=d_{N}=d_{P}=\tilde{d}$, then we get

$$
\begin{aligned}
\frac{\partial \Psi(u, t)}{\partial t}-\tilde{d} \Delta \Psi(u, t) & =\delta-\xi K(u, t)-\frac{\varrho}{2} L(u, t)-\frac{\varrho \eta}{2 \tau} M(u, t)-\frac{\varrho \Phi_{1} \alpha_{1}}{2 \tau \lambda_{1}} N(u, t)-\frac{\varrho \Phi_{2} \alpha_{2}}{2 \tau \lambda_{2}} \\
P(u, t) & \leq \delta-\sigma\left(K(u, t)+L(u, t)+\frac{\varrho}{2 \tau} M(u, t)+\frac{\varrho \Phi_{1}}{2 \tau \lambda_{1}} N(u, t)+\frac{\varrho \Phi_{2}}{2 \tau \lambda_{2}} P(u, t)\right) \\
& =\delta-\sigma \Psi(u, t),
\end{aligned}
$$


where $\sigma=\min \left\{\xi,(1 / 2) \varrho, \eta, \alpha_{1}, \alpha_{2}\right\}$. Thus, $\Psi(u, t)$ satisfies

$$
\left\{\begin{array}{l}
\frac{\partial \Psi(u, t)}{\partial t}-\tilde{d} \Delta \Psi(u, t) \leq \delta-\sigma \Psi(u, t) \\
\Psi(u, 0)=\mathscr{G}_{1}(u)+\mathscr{G}_{2}(u)+\frac{\varrho}{2 \tau} \mathscr{G}_{3}(u)+\frac{\varrho \Phi_{1}}{2 \tau \lambda_{1}} \mathscr{G}_{4}(u)+\frac{\varrho \Phi_{2}}{2 \tau \lambda_{2}} \mathscr{G}_{5}(u) \geq 0 \\
\frac{\partial \Psi}{\partial \overrightarrow{\mathscr{B}}}=0 .
\end{array}\right.
$$

Let $\widetilde{\Psi}(t)$ be a solution of the following ODE:

$$
\left\{\begin{array}{l}
\frac{\mathrm{d} \widetilde{\Psi}(t)}{\mathrm{d} t}=\delta-\sigma \widetilde{\Psi}(t), \\
\widetilde{\Psi}(0)=\max _{u \in \bar{\Gamma}} \Psi(u, 0) .
\end{array}\right.
$$

This gives that $\widetilde{\Psi}(t) \leq \max \left\{(\delta / \sigma), \max _{u \in \bar{\Gamma}} \Psi(u, 0)\right\}$. On the basis of comparison principle [38], we obtain $\Psi(u, t) \leq \widetilde{\Psi}(t)$. Hence,

$$
\Psi(u, t) \leq \max \left\{\frac{\delta}{\sigma}, \max _{u \in \bar{\Gamma}} \Psi(u, 0)\right\},
$$

which implies that $K(u, t), L(u, t), M(u, t), N(u, t)$, and $P(u, t)$ are bounded on $\bar{\Gamma} \times\left[0, \mathscr{T}_{m}\right)$. The standard theory for semilinear parabolic systems implies that $\mathscr{T}_{m}=+\infty$ [39]. This shows that solution $(K(u, t), L(u, t), M(u, t)$, $N(u, t), P(u, t))$ is defined for all $u \in \Gamma, t>0$, and also is unique and nonnegative.

\section{Equilibria}

Theorem 2. There exist three threshold parameters $\mathscr{R}_{0}, \mathscr{R}_{1}$, and $\mathscr{R}_{2}$ with $\mathscr{R}_{0}>\mathscr{R}_{1}$ and $\mathscr{R}_{0}>\mathscr{R}_{2}$, such that

(i) If $\mathscr{R}_{0} \leq 1$, then the system has a single equilibrium $\Omega_{0}$

(ii) If $\mathscr{R}_{1} \leq 1<\mathscr{R}_{0}$ and $\mathscr{R}_{2} \leq 1<\mathscr{R}_{0}$, then the system contains only two equilibria $\Omega_{0}$ and $\Omega_{1}$

(iii) If $\mathscr{R}_{1}>1$ and $\mathscr{R}_{2}<1$, then the system contains three equilibria $\Omega_{0}, \Omega_{1}$, and $\Omega_{2}$

(iv) If $\mathscr{R}_{2}>1$ and $\mathscr{R}_{1}<1$, then the system contains three equilibria $\Omega_{0}, \Omega_{1}$, and $\Omega_{3}$

(v) If $\mathscr{R}_{2}>1$ and $\mathscr{R}_{1}>1$, then the system contains four equilibria $\Omega_{0}, \Omega_{1}, \Omega_{2}$, and $\Omega_{3}$

Proof. To calculate the equilibria of systems (2)-(6), we let

$$
\begin{aligned}
& 0=\delta-\mu K M-\xi K, \\
& 0=\mu K M-\varrho L, \\
& 0=\tau L-\eta M-\varpi_{1} M N-\varpi_{2} M P, \\
& 0=\lambda_{1} M N-\alpha_{1} N, \\
& 0=\lambda_{2} M P-\alpha_{2} P .
\end{aligned}
$$

Then, solving the system of algebraic equations (18)-(22), we get four equilibria such as the following:

(i) Infection-free equilibrium $\Omega_{0}\left(K_{0}, 0,0,0,0,0\right)$, where $K_{0}=\delta / \xi$

(ii) Persistent DENV infection equilibrium with ineffective antibodies is $\Omega_{1}\left(K_{1}, L_{1}, M_{1}, 0,0\right)$, where

$$
\begin{aligned}
K_{1} & =\frac{K_{0}}{\mathscr{R}_{0}}, \\
L_{1} & =\frac{\eta \xi}{\tau \mu}\left(\mathscr{R}_{0}-1\right), \\
M_{1} & =\frac{\xi}{\mu}\left(\mathscr{R}_{0}-1\right) .
\end{aligned}
$$

(iii) Persistent DENV infection equilibrium with only effective heterologous antibody is $\Omega_{2}\left(K_{2}, L_{2}, M_{2}, N_{2}, 0\right)$, where

$$
\begin{aligned}
K_{2} & =\frac{\lambda_{1} \delta}{\xi \lambda_{1}+\mu \alpha_{1}}, \\
L_{2} & =\frac{\mu \delta \alpha_{1}}{\varrho\left(\xi \lambda_{1}+\mu \alpha_{1}\right)}, \\
M_{2} & =\frac{\alpha_{1}}{\lambda_{1}}, \\
N_{2} & =\frac{\eta}{\varpi_{1}}\left(\mathscr{R}_{1}-1\right) .
\end{aligned}
$$


(iv) Persistent DENV infection equilibrium with only effective homologous antibody is $\Omega_{3}\left(K_{3}, L_{3}, M_{3}, 0, P_{3}\right)$, where

$$
\begin{aligned}
K_{3} & =\frac{\lambda_{2} \delta}{\xi \lambda_{2}+\mu \alpha_{2}}, \\
L_{3} & =\frac{\mu \delta \alpha_{2}}{\varrho\left(\xi \lambda_{2}+\mu \alpha_{2}\right)}, \\
M_{3} & =\frac{\alpha_{2}}{\lambda_{2}}, \\
P_{3} & =\frac{\eta}{\omega_{2}}\left(\mathscr{R}_{2}-1\right) .
\end{aligned}
$$

Where

$$
\begin{aligned}
& \mathscr{R}_{0}=\frac{K_{0} \tau \mu}{\varrho \eta}, \\
& \mathscr{R}_{1}=\frac{\mathscr{R}_{0}}{1+\left(\mu \alpha_{1} / \xi \lambda_{1}\right)}, \\
& \mathscr{R}_{2}=\frac{\mathscr{R}_{0}}{1+\left(\mu \alpha_{2} / \xi \lambda_{2}\right)} .
\end{aligned}
$$

Clearly, $\mathscr{R}_{0}>\mathscr{R}_{1}$ and $\mathscr{R}_{0}>\mathscr{R}_{2}$ (v) Clearly from (iii) and (iv), if $\mathscr{R}_{1}>1$ and $\mathscr{R}_{2}>1$, then $\Omega_{0}, \Omega_{1}, \Omega_{2}$, and $\Omega_{3}$ all exist

Here, $\mathscr{R}_{0}$ represents the basic infection reproduction number, $\mathscr{R}_{1}$ represents the heterologous antibody immune response activation number, and $\mathscr{R}_{2}$ is the homologous antibody immune response activation number.

\section{Global Stability}

In this section, we investigate the global asymptotic stability of all equilibria by the Lyapunov method. The construction of Lyapunov functions are based on the works presented in [40-44]. To prove Theorems 1-4, we need to define a function $g(s)=s-1-\ln s$ and the arithmetic-geometric mean inequality:

$$
\frac{1}{s} \sum_{\ell=1}^{s} \chi_{\ell} \geq \sqrt{[n]} \prod_{\ell=1}^{s} \chi_{\ell}, \quad \chi_{\ell} \geq 0, \ell=1,2, \ldots,
$$

which implies

$$
\frac{1}{3}\left[\frac{K_{\ell}}{K}+\frac{L M_{\ell}}{L_{\ell} M}+\frac{M K L_{\ell}}{M_{\ell} K_{\ell} L}\right] \geq 1, \quad \ell=1,2,3 .
$$

Neumann boundary conditions (8) and divergence theorem imply that

$$
\begin{aligned}
& 0=\int_{\partial \Gamma} \nabla \mathscr{U} \cdot \overrightarrow{\mathscr{B}} \mathrm{d} u=\int_{\Gamma} \operatorname{div}(\nabla \mathcal{U}) \mathrm{d} u=\int_{\Gamma} \Delta \mathscr{U} \mathrm{d} u \\
& 0=\int_{\partial \Gamma} \frac{1}{\mathscr{U}} \nabla \mathscr{U} \cdot \overrightarrow{\mathscr{B}} \mathrm{d} u=\int_{\Gamma} \operatorname{div}\left(\frac{1}{\mathcal{U}} \nabla \mathcal{U}\right) \mathrm{d} u=\int_{\Gamma}\left(\frac{\Delta \mathcal{U}}{\mathcal{U}}-\frac{\|\nabla \mathscr{U}\|^{2}}{\mathcal{U}^{2}}\right) \mathrm{d} u, \\
& Y_{\ell}=\left\{(K, L, M, N, P): \frac{\mathrm{d} \widetilde{\Pi}_{\ell}}{\mathrm{d} t}=0\right\}, \quad \ell=0,1,2,3 .
\end{aligned}
$$

for $\mathcal{U} \in\{K, L, M, N, P\}$. Thus, we obtain

$$
\begin{aligned}
& \int_{\Gamma} \Delta \mathscr{U} \mathrm{d} u=0 \\
& \int_{\Gamma} \frac{\Delta \mathscr{U}}{\mathscr{U}} \mathrm{d} u=\int_{\Gamma} \frac{\|\nabla \mathscr{U}\|^{2}}{\mathcal{U}^{2}} \mathrm{~d} u .
\end{aligned}
$$

For convenience, we drop the input notation, i.e., $\quad(K, L, M, N, P)=(K(u, t), L(u, t), M(u, t), N(u, t), P$ $(u, t))$.

Consider a function $\Pi_{\ell}(K, L, M, N, P)$ and define

$$
\widetilde{\Pi}_{\ell}(t)=\int_{\Gamma} \Pi_{\ell}(u, t) \mathrm{d} u, \quad \ell=0,1,2,3 .
$$

Let $Y_{\ell}^{\prime}$ be the largest invariant subset of

Theorem 3. Let $\mathscr{R}_{0} \leq 1$, then $\Omega_{0}$ is globally asymptotically stable (GAS).

Proof. Define $\Pi_{0}(u, t)$ as

$$
\Pi_{0}(u, t)=K_{0} g\left(\frac{K}{K_{0}}\right)+L+\frac{\varrho}{\tau} M+\frac{\varrho \emptyset_{1}}{\tau \lambda_{1}} N+\frac{\varrho \Phi_{2}}{\tau \lambda_{2}} P .
$$

Clearly, $\widetilde{\Pi}_{0}(K, L, M, N, P)>0$ for all $(K, L, M, N, P)>0$ and $\widetilde{\Pi}_{0}\left(K_{0}, 0,0,0,0\right)=0$. We calculate $\partial \Pi_{0} / \partial t$ along the solutions of model (2)-(6) as 


$$
\begin{aligned}
\frac{\partial \Pi_{0}}{\partial t}= & \left(1-\frac{K_{0}}{K}\right)\left(d_{K} \Delta K+\delta-\xi K-\mu K M\right)+d_{L} \Delta L+\mu K M-\varrho L \\
& +\frac{\varrho}{\tau}\left(d_{M} \Delta M+\tau L-\eta M-\varpi_{1} M N-\varpi_{2} M P\right)+\frac{\varrho \varpi_{1}}{\tau \lambda_{1}}\left(d_{N} \Delta N+\lambda_{1} M N-\alpha_{1} N\right) \\
& +\frac{\varrho \varpi_{2}}{\tau \lambda_{2}}\left(d_{P} \Delta P+\lambda_{2} M P-\alpha_{2} P\right)
\end{aligned}
$$

Collecting terms of equation (34), we obtain

$$
\begin{aligned}
& \frac{\partial \Pi_{0}}{\partial t}=-\frac{\xi\left(K-K_{0}\right)}{K^{2}}+\left(\mu K_{0}-\frac{\varrho \eta}{\tau}\right) M-\frac{\varrho \varpi_{1} \alpha_{1}}{\tau \lambda_{1}} N-\frac{\varrho \varpi_{2} \alpha_{2}}{\tau \lambda_{2}} P \\
& +\left(1-\frac{K_{0}}{K}\right) d_{K} \Delta K+d_{L} \Delta L+\frac{\varrho}{\tau} d_{M} \Delta M+\frac{\varrho \varpi_{1}}{\tau \lambda_{1}} d_{N} \Delta N+\frac{\varrho \varpi_{2}}{\tau \lambda_{2}} d_{P} \Delta P \\
& =-\frac{\xi\left(K-K_{0}\right)}{K^{2}}+\frac{\varrho \eta}{\tau}\left(\mathscr{R}_{0}-1\right) M-\frac{\varrho \varpi_{1} \alpha_{1}}{\tau \lambda_{1}} N-\frac{\varrho \varpi_{2} \alpha_{2}}{\tau \lambda_{2}} P \\
& +\left(1-\frac{K_{0}}{K}\right) d_{K} \Delta K+d_{L} \Delta L+\frac{\varrho}{\tau} d_{M} \Delta M+\frac{\varrho \varpi_{1}}{\tau \lambda_{1}} d_{N} \Delta N+\frac{\varrho \varpi_{2}}{\tau \lambda_{2}} d_{P} \Delta P .
\end{aligned}
$$

Consequently, we calculate $\mathrm{d} \widetilde{\Pi}_{0} / \mathrm{d} t$ as follows:

$$
\begin{aligned}
\frac{\mathrm{d} \widetilde{\Pi}_{0}}{\mathrm{~d} t}= & -\xi \int_{\Gamma} \frac{\left(K-K_{0}\right)}{K^{2}} \mathrm{~d} u+\frac{\varrho \eta}{\tau}\left(\mathscr{R}_{0}-1\right) \int_{\Gamma} M \mathrm{~d} u-\frac{\varrho \varpi_{1} \alpha_{1}}{\tau \lambda_{1}} \int_{\Gamma} N \mathrm{~d} u-\frac{\varrho \Phi_{2} \alpha_{2}}{\tau \lambda_{2}} \int_{\Gamma} P \mathrm{~d} u \\
& +d_{K} \int_{\Gamma}\left(1-\frac{K_{0}}{K}\right) \Delta K \mathrm{~d} u+d_{L} \int_{\Gamma} \Delta L \mathrm{~d} u+\frac{\varrho}{\tau} d_{M} \int_{\Gamma} \Delta M \mathrm{~d} u \\
& +\frac{\varrho \emptyset_{1}}{\tau \lambda_{1}} d_{N} \int_{\Gamma} \Delta N \mathrm{~d} u+\frac{\varrho \Phi_{2}}{\tau \lambda_{2}} d_{P} \int_{\Gamma} \Delta P \mathrm{~d} u .
\end{aligned}
$$

Using equality (30), equation (36) is reduced to the following form:

$$
\begin{aligned}
\frac{\mathrm{d} \widetilde{\Pi}_{0}}{\mathrm{~d} t}= & -\xi \int_{\Gamma} \frac{\left(K-K_{0}\right)}{K^{2}} \mathrm{~d} u+\frac{\varrho \eta}{\tau}\left(\mathscr{R}_{0}-1\right) \int_{\Gamma} M \mathrm{~d} u \\
& -\frac{\varrho \varpi_{1} \alpha_{1}}{\tau \lambda_{1}} \int_{\Gamma} N \mathrm{~d} u \\
& -\frac{\varrho \varpi_{2} \alpha_{2}}{\tau \lambda_{2}} \int_{\Gamma} P \mathrm{~d} u-d_{K} K_{0} \int_{\Gamma} \frac{\|\nabla K\|^{2}}{K^{2}} \mathrm{~d} u .
\end{aligned}
$$

Therefore, $\left(\mathrm{d} \widetilde{\Pi}_{0} / \mathrm{d} t\right) \leq 0$, for all $K, M, N, P>0$, and $\left(\mathrm{d} \widetilde{\Pi}_{0} / \mathrm{d} t\right)=0$ with equality holding when $(K, M, N, P)=\left(K_{0}, 0,0,0\right)$. The solutions of models $(2)-(6)$ converge to $Y_{0}^{\prime}$. The elements of $Y_{0}^{\prime}$ satisfy $(K, M, N, P)=\left(K_{0}, 0,0,0\right)$, and then, $(\partial M / \partial t)=\Delta M=0$. Equation (6) reduces to

$$
0=\frac{\partial M}{\partial t}=\tau L
$$

This yields $L=0$. Hence, $Y_{0}^{\prime}=\left\{\Omega_{0}\right\}$, and by applying LLIP [45-47], we get that $\Omega_{0}$ is GAS.

Theorem 4. If $\mathscr{R}_{1} \leq 1<\mathscr{R}_{0}$ and $\mathscr{R}_{2} \leq 1$, then $\Omega_{1}$ is GAS.

Proof. Define $\Pi_{1}(u, t)$ as

$$
\begin{aligned}
\Pi_{1}(u, t)= & K_{1} g\left(\frac{K}{K_{1}}\right)+L_{1} g\left(\frac{L}{L_{1}}\right)+\frac{\varrho}{\tau} M_{1} g\left(\frac{M}{M_{1}}\right) \\
& +\frac{\varrho \emptyset_{1}}{\tau \lambda_{1}} N+\frac{\varrho \emptyset_{2}}{\tau \lambda_{2}} P .
\end{aligned}
$$

Calculate $\partial \Pi_{1} / \partial t$ as 


$$
\begin{aligned}
& \frac{\partial \Pi_{1}}{\partial t}=\left(1-\frac{K_{1}}{K}\right)\left(d_{K} \Delta K+\delta-\xi K-\mu K M\right)+\left(1-\frac{L_{1}}{L}\right)\left(d_{L} \Delta L+\mu K M-\varrho L\right) \\
& +\frac{\varrho}{\tau}\left(1-\frac{M_{1}}{M}\right)\left(d_{M} \Delta M+\tau L-\eta M-\varpi_{1} M N-\varpi_{2} M P\right) \\
& +\frac{\varrho \varpi_{1}}{\tau \lambda_{1}}\left(d_{N} \Delta N+\lambda_{1} M N-\alpha_{1} N\right)+\frac{\varrho \varpi_{2}}{\tau \lambda_{2}}\left(d_{P} \Delta P+\lambda_{2} M P-\alpha_{2} P\right) \\
& =\left(1-\frac{K_{1}}{K}\right)(\delta-\xi K)+\mu K_{1} M-\frac{\mu K M L_{1}}{L}+\varrho L_{1}-\frac{\varrho \eta}{\tau} M-\varrho \frac{M_{1} L}{M} \\
& +\frac{\varrho \eta}{\tau} M_{1}+\frac{\varrho \Phi_{1}}{\tau} M_{1} N+\frac{\varrho \varpi_{2}}{\tau} M_{1} P-\frac{\varrho \Phi_{1} \alpha_{1}}{\tau \lambda_{1}} N-\frac{\varrho \varpi_{2} \alpha_{2}}{\tau \lambda_{2}} P \\
& +d_{K}\left(1-\frac{K_{1}}{K}\right) \Delta K+d_{L}\left(1-\frac{L_{1}}{L}\right) \Delta L+\frac{\varrho d_{M}}{\tau}\left(1-\frac{M_{1}}{M}\right) \Delta M \\
& +\frac{\varrho \varpi_{1} d_{N}}{\tau \lambda_{1}} \Delta N+\frac{\varrho \varpi_{2} d_{P}}{\tau \lambda_{2}} \Delta P
\end{aligned}
$$

The equilibrium conditions of $\Omega_{1}$ imply that

$$
\begin{aligned}
\delta & =\xi K_{1}+\mu K_{1} M_{1}, \\
\varrho L_{1} & =\mu K_{1} M_{1}, \\
\tau L_{1} & =\eta M_{1},
\end{aligned}
$$

$$
\begin{aligned}
\frac{\partial \Pi_{1}}{\partial t}= & -\frac{\xi\left(K-K_{1}\right)^{2}}{K}+\varrho L_{1}\left(1-\frac{K_{1}}{K}\right)-\varrho L_{1} \frac{K M L_{1}}{K_{1} M_{1} L}+2 \varrho L_{1}-\varrho L_{1} \frac{M_{1} L}{M L_{1}} \\
& +\frac{\varrho \Phi_{1}}{\tau}\left(M_{1}-\frac{\alpha_{1}}{\lambda_{1}}\right) N+\frac{\varrho \varpi_{2}}{\tau}\left(M_{1}-\frac{\alpha_{2}}{\lambda_{2}}\right) P+d_{K}\left(1-\frac{K_{1}}{K}\right) \Delta K+d_{L}\left(1-\frac{L_{1}}{L}\right) \Delta L \\
& +\frac{\varrho d_{M}}{\tau}\left(1-\frac{M_{1}}{M}\right) \Delta M+\frac{\varrho \Phi_{1} d_{N}}{\tau \lambda_{1}} \Delta N+\frac{\varrho \Phi_{2} d_{P}}{\tau \lambda_{2}} \Delta P=-\frac{\xi\left(K-K_{1}\right)^{2}}{K} \\
& +\varrho L_{1}\left[3-\frac{K_{1}}{K}-\frac{K M L_{1}}{K_{1} M_{1} L}-\frac{M_{1} L}{M L_{1}}\right]+\frac{\varrho \varpi_{1}\left(\xi \lambda_{1}+\mu \alpha_{1}\right)}{\tau \lambda_{1} \mu}\left(\mathscr{R}_{1}-1\right) N \\
& +\frac{\varrho \Phi_{2}\left(\xi \lambda_{2}+\mu \alpha_{2}\right)}{\tau \lambda_{2} \mu}\left(\mathscr{R}_{2}-1\right) P+d_{K}\left(1-\frac{K_{1}}{K}\right) \Delta K+d_{L}\left(1-\frac{L_{1}}{L}\right) \Delta L+\frac{\varrho d_{M}}{\tau}\left(1-\frac{M_{1}}{M}\right) \Delta M \\
& +\frac{\varrho \Phi_{1} d_{N}}{\tau \lambda_{1}} \Delta N+\frac{\varrho \Phi_{2} d_{P}}{\tau \lambda_{2}} \Delta P .
\end{aligned}
$$


Calculate the time derivative of $\widetilde{\Pi}_{1}(t)$ and use equality (30) to get

$$
\begin{aligned}
& \frac{\mathrm{d} \widetilde{\Pi}_{1}}{\mathrm{~d} t}=-\xi \int_{\Gamma} \frac{\left(K-K_{1}\right)^{2}}{K} \mathrm{~d} u+\varrho L_{1} \int_{\Gamma}\left[3-\frac{K_{1}}{K}-\frac{K M L_{1}}{K_{1} M_{1} L}-\frac{M_{1} L}{M L_{1}}\right] \mathrm{d} u \\
& +\frac{\varrho \Phi_{1}\left(\xi \lambda_{1}+\mu \alpha_{1}\right)}{\tau \lambda_{1} \mu}\left(\mathscr{R}_{1}-1\right) \int_{\Gamma} N \mathrm{~d} u+\frac{\varrho \omega_{2}\left(\xi \lambda_{2}+\mu \alpha_{2}\right)}{\tau \lambda_{2} \mu}\left(\mathscr{R}_{2}-1\right) \int_{\Gamma} P \mathrm{~d} u \\
& +d_{K} \int_{\Gamma}\left(1-\frac{K_{1}}{K}\right) \Delta K \mathrm{~d} u+d_{L} \int_{\Gamma}\left(1-\frac{L_{1}}{L}\right) \Delta L \mathrm{~d} u+\frac{\varrho d_{M}}{\tau} \int_{\Gamma}\left(1-\frac{M_{1}}{M}\right) \Delta M \mathrm{~d} u \\
& +\frac{\varrho \varpi_{1} d_{N}}{\tau \lambda_{1}} \int_{\Gamma} \Delta N \mathrm{~d} u+\frac{\varrho \varpi_{2} d_{P}}{\tau \lambda_{2}} \int_{\Gamma} \Delta P \mathrm{~d} u=-\xi \int_{\Gamma} \frac{\left(K-K_{1}\right)^{2}}{K} \mathrm{~d} u \\
& +\varrho L_{1} \int_{\Gamma}\left[3-\frac{K_{1}}{K}-\frac{K M L_{1}}{K_{1} M_{1} L}-\frac{M_{1} L}{M L_{1}}\right] \mathrm{d} u+\frac{\varrho \Phi_{1}\left(\xi \lambda_{1}+\mu \alpha_{1}\right)}{\tau \lambda_{1} \mu}\left(\mathscr{R}_{1}-1\right) \int_{\Gamma} N \mathrm{~d} u \\
& +\frac{\varrho \varpi_{2}\left(\xi \lambda_{2}+\mu \alpha_{2}\right)}{\tau \lambda_{2} \mu}\left(\mathscr{R}_{2}-1\right) \int_{\Gamma} P \mathrm{~d} u-d_{K} K_{1} \int_{\Gamma} \frac{\|\nabla K\|^{2}}{K^{2}} \mathrm{~d} u-d_{L} L_{1} \int_{\Gamma} \frac{\|\nabla L\|^{2}}{L^{2}} \mathrm{~d} u \\
& -\frac{\varrho d_{M}}{\tau} M_{1} \int_{\Gamma} \frac{\|\nabla M\|^{2}}{M^{2}} \mathrm{~d} u .
\end{aligned}
$$

Since $\mathscr{R}_{1} \leq 1$ and $\mathscr{R}_{2} \leq 1$, then utilizing inequality (28), we obtain $\mathrm{d} \widetilde{\Pi}_{1} / \mathrm{d} t \leq 0$ for all $K, L, M, N, P>0$. Furthermore, $\mathrm{d} \widetilde{\Pi}_{1} / \mathrm{d} t=0$ at $(K, L, M, N, P)=\left(K_{1}, L_{1}, M_{1}, 0,0\right)$. The solutions of (2)-(6) tend to $Y_{1}^{\prime}=\left\{\Omega_{1}\right\}$. It follows that $\Omega_{1}$ is GAS by using LLIP.

Theorem 5. Let $\mathscr{R}_{1}>1$ and $\mathscr{R}_{2} \leq \mathscr{R}_{1}$ then $\Omega_{2}$ is GAS.

$$
\begin{aligned}
\Pi_{2}(u, t)= & K_{2} g\left(\frac{K}{K_{2}}\right)+L_{2} g\left(\frac{L}{L_{2}}\right)+\frac{\varrho}{\tau} M_{2} g\left(\frac{M}{M_{2}}\right) \\
& +\frac{\varrho \Phi_{1}}{\tau \lambda_{1}} N_{2} g\left(\frac{N}{N_{2}}\right)+\frac{\varrho \Phi_{2}}{\tau \lambda_{2}} P .
\end{aligned}
$$

Calculate $\partial \Pi_{2} / \partial t$ as

Proof. Define $\Pi_{2}(u, t)$ as

$$
\begin{aligned}
\frac{\partial \Pi_{2}}{\partial t}= & \left(1-\frac{K_{2}}{K}\right)\left(d_{K} \Delta K+\delta-\xi K-\mu K M\right)+\left(1-\frac{L_{2}}{L}\right)\left(d_{L} \Delta L+\mu K M-\varrho L\right) \\
& +\frac{\varrho}{\tau}\left(1-\frac{M_{2}}{M}\right)\left(d_{M} \Delta M+\tau L-\eta M-\varpi_{1} M N-\varpi_{2} M P\right) \\
& +\frac{\varrho \varpi_{1}}{\tau \lambda_{1}}\left(1-\frac{N_{2}}{N}\right)\left(d_{N} \Delta N+\lambda_{1} M N-\alpha_{1} N\right)+\frac{\varrho \varpi_{2}}{\tau \lambda_{2}}\left(d_{P} \Delta P+\lambda_{2} M P-\alpha_{2} P\right) .
\end{aligned}
$$

Collecting terms of equation (45), we get

$$
\begin{aligned}
\frac{\partial \Pi_{2}}{\partial t}= & \left(1-\frac{K_{2}}{K}\right)(\delta-\xi K)+\mu K_{2} M-\frac{\mu K M L_{2}}{L}+\varrho L_{2}-\frac{\varrho \eta}{\tau} M-\varrho \frac{M_{2} L}{M}+\frac{\varrho \eta}{\tau} M_{2} \\
& +\frac{\varrho \Phi_{1}}{\tau} M_{2} N+\frac{\varrho \Phi_{2}}{\tau} M_{2} P-\frac{\varrho \Phi_{1} \alpha_{1}}{\tau \lambda_{1}} N-\frac{\varrho \Phi_{1}}{\tau} N_{2} M+\frac{\varrho \Phi_{1} \alpha_{1}}{\tau \lambda_{1}} N_{2}-\frac{\varrho \Phi_{2} \alpha_{2}}{\tau \lambda_{2}} P \\
& +d_{K}\left(1-\frac{K_{2}}{K}\right) \Delta K+d_{L}\left(1-\frac{L_{2}}{L}\right) \Delta L+\frac{\varrho d_{M}}{\tau}\left(1-\frac{M_{2}}{M}\right) \Delta M+\frac{\varrho \varpi_{1} d_{N}}{\tau \lambda_{1}}\left(1-\frac{N_{2}}{N}\right) \Delta N
\end{aligned}
$$




$$
\begin{aligned}
& +\frac{\varrho \emptyset_{2} d_{P}}{\tau \lambda_{2}} \Delta P=\left(1-\frac{K_{2}}{K}\right)(\delta-\xi K)+\left(\mu K_{2}-\frac{\varrho \eta}{\tau}-\frac{\varrho \varpi_{1}}{\tau} N_{2}\right) M+\frac{\varrho \varpi_{1}}{\tau}\left(M_{2}-\frac{\alpha_{1}}{\lambda_{1}}\right) N \\
& -\frac{\mu K M L_{2}}{L}+\varrho L_{2}-\varrho \frac{M_{2} L}{M}+\frac{\varrho \eta}{\tau} M_{2}+\frac{\varrho \Phi_{2}}{\tau} M_{2} P+\frac{\varrho \Phi_{1} \alpha_{1}}{\tau \lambda_{1}} N_{2}-\frac{\varrho \Phi_{2} \alpha_{2}}{\tau \lambda_{2}} P \\
& +d_{K}\left(1-\frac{K_{2}}{K}\right) \Delta K+d_{L}\left(1-\frac{L_{2}}{L}\right) \Delta L+\frac{\varrho d_{M}}{\tau}\left(1-\frac{M_{2}}{M}\right) \Delta M \\
& +\frac{\varrho \varpi_{1} d_{N}}{\tau \lambda_{1}}\left(1-\frac{N_{2}}{N}\right) \Delta N+\frac{\varrho \varpi_{2} d_{P}}{\tau \lambda_{2}} \Delta P
\end{aligned}
$$

Applying the equilibrium conditions,

$$
\begin{aligned}
\delta & =\xi K_{2}+\mu K_{2} M_{2}, \\
\varrho L_{2} & =\mu K_{2} M_{2}, \\
\tau L_{2} & =\eta M_{2}+\varpi_{1} M_{2} N_{2}, \\
M_{2} & =\frac{\alpha_{1}}{\lambda_{1}},
\end{aligned}
$$

$$
\begin{aligned}
\frac{\partial \Pi_{2}}{\partial t}= & -\frac{\xi\left(K-K_{2}\right)^{2}}{K}+\varrho L_{2}\left(1-\frac{K_{2}}{K}\right)-\varrho L_{2} \frac{K M L_{2}}{K_{2} M_{2} L}-\varrho L_{2} \frac{M_{2} L}{M L_{2}}+2 \varrho L_{2}+\frac{\varrho \emptyset_{2}}{\tau} \\
& \cdot\left(M_{2}-M_{3}\right) P+d_{K}\left(1-\frac{K_{2}}{K}\right) \Delta K+d_{L}\left(1-\frac{L_{2}}{L}\right) \Delta L+\frac{\varrho d_{M}}{\tau}\left(1-\frac{M_{2}}{M}\right) \Delta M+\frac{\varrho \Phi_{1} d_{N}}{\tau \lambda_{1}} \\
& \cdot\left(1-\frac{N_{2}}{N}\right) \Delta N+\frac{\varrho \Phi_{2} d_{P}}{\tau \lambda_{2}} \Delta P=-\frac{\xi\left(K-K_{2}\right)^{2}}{K}+\varrho L_{2}\left[3-\frac{K_{2}}{K}-\frac{K M L_{2}}{K_{2} M_{2} L}-\frac{M_{2} L}{M L_{2}}\right] \\
& +\frac{\varrho \Phi_{2}\left(\xi \lambda_{2}+\mu \alpha_{2}\right)}{\tau \mu \lambda_{2} \mathscr{R}_{1}}\left(\mathscr{R}_{2}-\mathscr{R}_{1}\right) P+d_{K}\left(1-\frac{K_{2}}{K}\right) \Delta K+d_{L}\left(1-\frac{L_{2}}{L}\right) \Delta L \\
& +\frac{\varrho d_{M}}{\tau}\left(1-\frac{M_{2}}{M}\right) \Delta M+\frac{\varrho \Phi_{1} d_{N}}{\tau \lambda_{1}}\left(1-\frac{N_{2}}{N}\right) \Delta N+\frac{\varrho \Phi_{2} d_{P}}{\tau \lambda_{2}} \Delta P .
\end{aligned}
$$

Calculate the time derivative of $\widetilde{\Pi}_{2}(t)$ and use equality (30) to get

$$
\begin{aligned}
\frac{\mathrm{d} \widetilde{\Pi}_{2}}{\mathrm{~d} t}= & -\xi \int_{\Gamma} \frac{\left(K-K_{2}\right)^{2}}{K} \mathrm{~d} u+\varrho L_{2} \int_{\Gamma}\left[3-\frac{K_{2}}{K}-\frac{K M L_{2}}{K_{2} M_{2} L}-\frac{M_{2} L}{M L_{2}}\right] \mathrm{d} u+\frac{\varrho \Phi_{2}\left(\xi \lambda_{2}+\mu \alpha_{2}\right)}{\tau \mu \lambda_{2} \mathscr{R}_{1}} \\
& \cdot\left(\mathscr{R}_{2}-\mathscr{R}_{1}\right) \int_{\Gamma} P \mathrm{~d} u+d_{K} \int_{\Gamma}\left(1-\frac{K_{2}}{K}\right) \Delta K \mathrm{~d} u+d_{L} \int_{\Gamma}\left(1-\frac{L_{2}}{L}\right) \Delta L \mathrm{~d} u+\frac{\varrho d_{M}}{\tau} \int_{\Gamma}\left(1-\frac{M_{2}}{M}\right) \Delta M \mathrm{~d} u \\
& +\frac{\varrho \Phi_{1} d_{N}}{\tau \lambda_{1}} \int_{\Gamma}\left(1-\frac{N_{2}}{N}\right) \Delta N \mathrm{~d} u+\frac{\varrho \emptyset_{2} d_{P}}{\tau \lambda_{2}} \int_{\Gamma} \Delta P \mathrm{~d} u=-\xi \int_{\Gamma} \frac{\left(K-K_{2}\right)^{2}}{K} \mathrm{~d} u
\end{aligned}
$$




$$
\begin{aligned}
& +\varrho L_{2} \int_{\Gamma}\left[3-\frac{K_{2}}{K}-\frac{K M L_{2}}{K_{2} M_{2} L}-\frac{M_{2} L}{M L_{2}}\right] \mathrm{d} u+\frac{\varrho \varrho_{2}\left(\xi \lambda_{2}+\mu \alpha_{2}\right)}{\tau \mu \lambda_{2} \mathscr{R}_{1}}\left(\mathscr{R}_{2}-\mathscr{R}_{1}\right) \int_{\Gamma} P \mathrm{~d} u \\
& -d_{K} K_{2} \int_{\Gamma} \frac{\|\nabla K\|^{2}}{K^{2}} \mathrm{~d} u-d_{L} L_{2} \int_{\Gamma} \frac{\|\nabla L\|^{2}}{L^{2}} \mathrm{~d} u-\frac{\varrho d_{M}}{\tau} M_{2} \int_{\Gamma} \frac{\|\nabla M\|^{2}}{M^{2}} \mathrm{~d} u-\frac{\varrho \Phi_{1} d_{N}}{\tau \lambda_{1}} N_{2} \int_{\Gamma} \frac{\|\nabla N\|^{2}}{N^{2}} \mathrm{~d} u .
\end{aligned}
$$

Since $\mathscr{R}_{2} \leq \mathscr{R}_{1}$, then using inequality (28), we obtain $\mathrm{d} \widetilde{\Pi}_{2} / \mathrm{d} t \leq 0$ for all $(K, L, M, N, P)>0$. Furthermore, $\mathrm{d} \widetilde{\Pi}_{2} / \mathrm{d} t \leq 0$ at $(K, L, M, N, P)=\left(K_{2}, L_{2}, M_{2}, N_{2}, 0\right)$. The solutions of (2)-(6) tend to $Y_{2}^{\prime}=\left\{\Omega_{2}\right\}$. It follows that $\Omega_{2}$ is GAS by using LLIP.

Theorem 6. Let $\mathscr{R}_{2}>1$ and $\mathscr{R}_{1} \leq \mathscr{R}_{2}$, then $\Omega_{3}$ is GAS.

Proof. Define $\Pi_{3}(u, t)$ as

$$
\begin{aligned}
\Pi_{3}(u, t)= & K_{3} g\left(\frac{K}{K_{3}}\right)+L_{3} g\left(\frac{L}{L_{3}}\right)+\frac{\varrho}{\tau} M_{3} g\left(\frac{M}{M_{3}}\right) \\
& +\frac{\varrho \Phi_{1}}{\tau \lambda_{1}} N+\frac{\varrho \Phi_{2}}{\tau \lambda_{2}} P_{3} g\left(\frac{P}{P_{3}}\right) .
\end{aligned}
$$
we get
Calculating $\partial \Pi_{3} / \partial t$ along the solutions of models (2)-(6),

$$
\begin{aligned}
\frac{\partial \Pi_{3}}{\partial t}= & \left(1-\frac{K_{3}}{K}\right)\left(d_{K} \Delta K+\delta-\xi K-\mu K M\right)+\left(1-\frac{L_{3}}{L}\right)\left(d_{L} \Delta L+\mu K M-\varrho L\right) \\
& +\frac{\varrho}{\tau}\left(1-\frac{M_{3}}{M}\right)\left(d_{M} \Delta M+\tau L-\eta M-\varpi_{1} M N-\omega_{2} M P\right) \\
& +\frac{\varrho \varpi_{1}}{\tau \lambda_{1}}\left(d_{N} \Delta N+\lambda_{1} M N-\alpha_{1} N\right)+\frac{\varrho \omega_{2}}{\tau \lambda_{2}}\left(1-\frac{P_{3}}{P}\right)\left(d_{P} \Delta P+\lambda_{2} M P-\alpha_{2} P\right) .
\end{aligned}
$$

Collecting terms, we get

$$
\begin{aligned}
& \frac{\partial \Pi_{3}}{\partial t}=\left(1-\frac{K_{3}}{K}\right)(\delta-\xi K)+\mu K_{3} M-\frac{\mu K M L_{3}}{L}+\varrho L_{3}-\frac{\varrho \eta}{\tau} M-\varrho \frac{M_{3} L}{M} \\
& +\frac{\varrho \eta}{\tau} M_{3}+\frac{\varrho ळ_{1}}{\tau} M_{3} N+\frac{\varrho \varrho_{2}}{\tau} M_{3} P-\frac{\varrho \varpi_{1} \alpha_{1}}{\tau \lambda_{1}} N-\frac{\varrho ळ_{2} \alpha_{2}}{\tau \lambda_{2}} P \\
& -\frac{\varrho \omega_{2}}{\tau} P_{3} M+\frac{\varrho \varpi_{2} \alpha_{2}}{\tau \lambda_{2}} P_{3}+d_{K}\left(1-\frac{K_{3}}{K}\right) \Delta K+d_{L}\left(1-\frac{L_{3}}{L}\right) \Delta L \\
& +\frac{\varrho d_{M}}{\tau}\left(1-\frac{M_{3}}{M}\right) \Delta M+\frac{\varrho \varpi_{1} d_{N}}{\tau \lambda_{1}} \Delta N+\frac{\varrho \varpi_{2} d_{P}}{\tau \lambda_{2}}\left(1-\frac{P_{3}}{P}\right) \Delta P .
\end{aligned}
$$

Applying the equilibrium conditions of $\Omega_{3}$,

$$
\begin{aligned}
\delta & =\xi K_{3}+\mu K_{3} M_{3}, \\
\varrho L_{3} & =\mu K_{3} M_{3}, \\
\tau L_{3} & =\eta M_{3}+\oplus_{2} M_{3} P_{3}, \\
\alpha_{2} P_{3} & =\lambda_{2} M_{3} P_{3},
\end{aligned}
$$


we get

$$
\begin{aligned}
\frac{\partial \Pi_{3}}{\partial t}= & -\frac{\xi\left(K-K_{3}\right)^{2}}{K}+\varrho L_{3}\left(1-\frac{K_{3}}{K}\right)-\varrho L_{3} \frac{K L_{3}}{K_{3} M_{3} L}-\varrho L_{3} \frac{M_{3} L}{M L_{3}}+2 \varrho L_{3} \\
& +\frac{\varrho \Phi_{1}}{\tau}\left(M_{3}-M_{2}\right) N+d_{K}\left(1-\frac{K_{3}}{K}\right) \Delta K+d_{L}\left(1-\frac{L_{3}}{L}\right) \Delta L+\frac{\varrho d_{M}}{\tau}\left(1-\frac{M_{3}}{M}\right) \Delta M \\
& +\frac{\varrho \varpi_{1} d_{N}}{\tau \lambda_{1}} \Delta N+\frac{\varrho \Phi_{2} d_{P}}{\tau \lambda_{2}}\left(1-\frac{P_{3}}{P}\right) \Delta P=-\frac{\xi\left(K-K_{3}\right)^{2}}{K}+\varrho L_{3}\left[3-\frac{K_{3}}{K}-\frac{K M L_{3}}{K_{3} M_{3} L}-\frac{M_{3} L}{M L_{3}}\right] \\
& +\frac{\varrho \varpi_{1}\left(\xi \lambda_{2}+\mu \alpha_{2}\right)}{\tau \mu \lambda_{2} \mathscr{R}_{1}}\left(\mathscr{R}_{1}-\mathscr{R}_{2}\right) N+d_{K}\left(1-\frac{K_{3}}{K}\right) \Delta K+d_{L}\left(1-\frac{L_{3}}{L}\right) \Delta L \\
& +\frac{\varrho d_{M}}{\tau}\left(1-\frac{M_{3}}{M}\right) \Delta M+\frac{\varrho \Phi_{1} d_{N}}{\tau \lambda_{1}} \Delta N+\frac{\varrho \varpi_{2} d_{P}}{\tau \lambda_{2}}\left(1-\frac{P_{3}}{P}\right) \Delta P .
\end{aligned}
$$

Calculating the time derivative of $\widetilde{\Pi}_{3}(t)$ and using equality (30), we obtain

$$
\begin{aligned}
\frac{\mathrm{d} \widetilde{\Pi}_{3}}{\mathrm{~d} t}= & \left.-\xi \int_{\Gamma} \frac{\left(K-K_{3}\right)^{2}}{K} \mathrm{~d} u+\varrho L_{3} \int_{\Gamma} \int_{\Gamma}-\frac{K_{3}}{K}-\frac{K M L_{3}}{K_{3} M_{3} L}-\frac{M_{3} L}{M L_{3}}\right] \mathrm{d} u+\frac{\varrho \Phi_{1}\left(\xi \lambda_{2}+\mu \alpha_{2}\right)}{\tau \mu \lambda_{2} \mathscr{R}_{1}} \\
& \cdot\left(\mathscr{R}_{1}-\mathscr{R}_{2}\right) \int_{\Gamma} N \mathrm{~d} u+d_{K} \int_{\Gamma}\left(1-\frac{K_{3}}{K}\right) \Delta K \mathrm{~d} u+d_{L} \int_{\Gamma}\left(1-\frac{L_{3}}{L}\right) \Delta L \mathrm{~d} u+\frac{\varrho d_{M}}{\tau} \int_{\Gamma}\left(1-\frac{M_{3}}{M}\right) \Delta M \mathrm{~d} u \\
& +\frac{\varrho \Phi_{1} d_{N}}{\tau \lambda_{1}} \int_{\Gamma} \Delta N \mathrm{~d} u+\frac{\varrho \omega_{2} d_{P}}{\tau \lambda_{2}} \int_{\Gamma}\left(1-\frac{P_{3}}{P}\right) \Delta P \mathrm{~d} u=-\xi \int_{\Gamma} \frac{\left(K-K_{3}\right)^{2}}{K} \mathrm{~d} u \\
& +\varrho L_{3} \int_{\Gamma}\left[3-\frac{K_{3}}{K}-\frac{K M L_{3}}{K_{3} M_{3} L}-\frac{M_{3} L}{M L_{3}}\right] \mathrm{d} u+\frac{\varrho \Phi_{1}\left(\xi \lambda_{2}+\mu \alpha_{2}\right)}{\tau \mu \lambda_{2} \mathscr{R}_{1}}\left(\mathscr{R}_{1}-\mathscr{R}_{2}\right) \\
& \cdot \int_{\Gamma} N \mathrm{~d} u-d_{K} K_{3} \int_{\Gamma} \frac{\|\nabla K\|^{2}}{K^{2}} \mathrm{~d} u-d_{L} L_{3} \int_{\Gamma} \frac{\|\nabla L\|^{2}}{L^{2}} \mathrm{~d} u-\frac{\varrho d_{M} M_{3}}{\tau} \int_{\Gamma} \frac{\|\nabla M\|^{2}}{M^{2}} \mathrm{~d} u \\
& -\frac{\varrho \Phi_{2} d_{P} P_{3}}{\tau \lambda_{2}} \int_{\Gamma} \frac{\|\nabla P\|^{2}}{P^{2}} \mathrm{~d} u .
\end{aligned}
$$

Since $\mathscr{R}_{1} \leq \mathscr{R}_{2}$, then using inequality (28), we obtain $\mathrm{d} \widetilde{\Pi}_{3} / \mathrm{d} t \leq 0$, for all $(K, L, M, N, P)>0$. Furthermore, $\mathrm{d} \widetilde{\Pi}_{3} / \mathrm{d} t \leq 0$ at $(K, L, M, N, P)=\left(K_{3}, L_{3}, M_{3}, 0, P_{3}\right)$. The solutions of (2)-(6) tend to $Y_{3}^{\prime}=\left\{\Omega_{3}\right\}$. It follows that $\Omega_{3}$ is GAS by using LLIP.

\section{Numerical Simulations}

In this section, we numerically illustrate the global stability of equilibria by choosing the domain $\Gamma$ as $\Gamma=[0,2]$ with a step size 0.02 . The step size for time is given by 0.1 . Following the works presented in $[31,48-50]$, we consider the following initial conditions for systems (2)-(6):

$$
\begin{aligned}
K(u, 0) & =500\left[1+0.4 \cos ^{2}(\pi u)\right], \\
L(u, 0) & =30\left[1+0.5 \cos ^{2}(\pi u)\right], \\
M(u, 0) & =4\left[1+0.5 \cos ^{2}(\pi u)\right], \\
N(u, 0) & =2\left[1+0.5 \cos ^{2}(\pi u)\right], \\
P(u, 0) & =2\left[1+0.5 \cos ^{2}(\pi u)\right], \quad u \in[0,2] .
\end{aligned}
$$

The initial values are arbitrarily chosen as the global stability of the equilibria presented in Theorems 3-6 guarantees the convergence regardless of the selected initial conditions.

In addition, we consider the following homogeneous Neumann boundary conditions: 


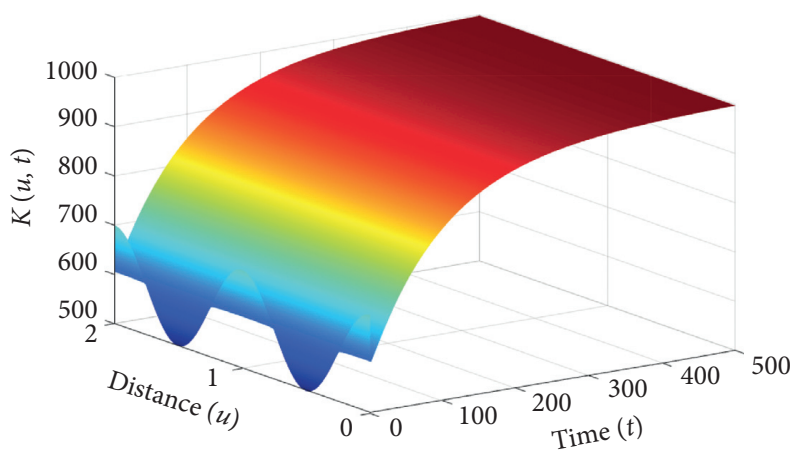

(a)

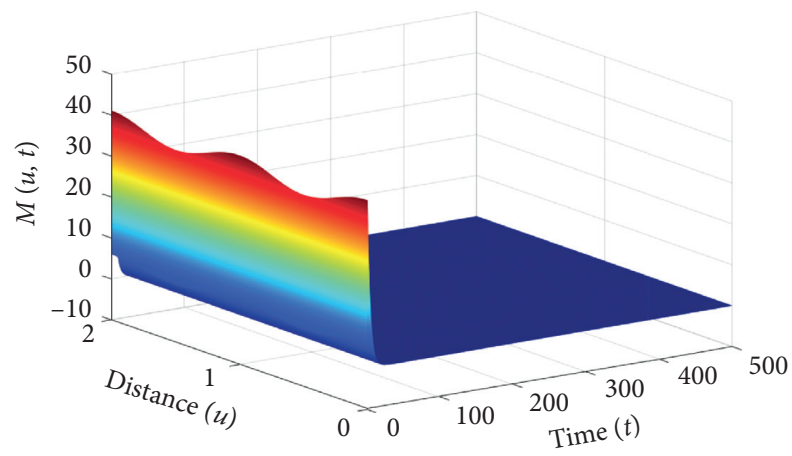

(c)

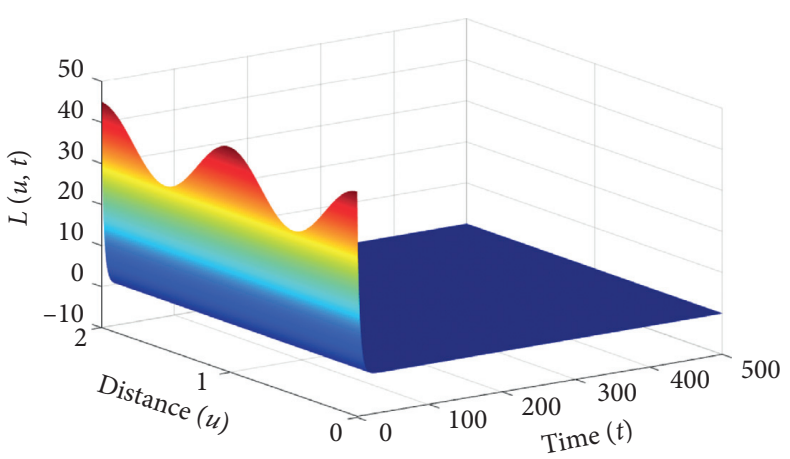

(b)

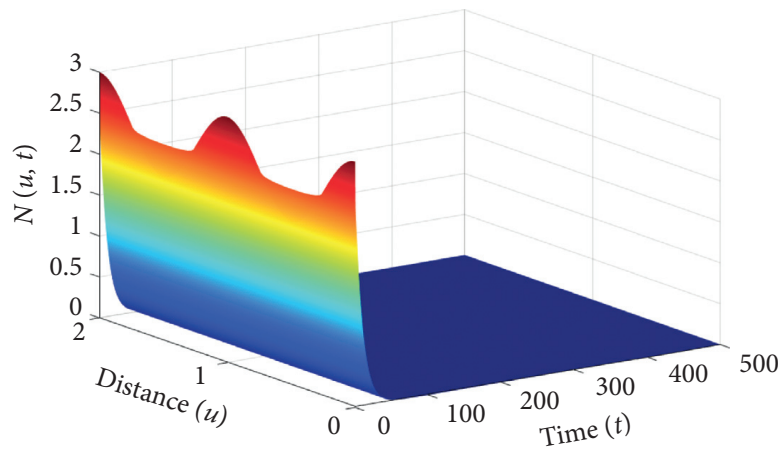

(d)

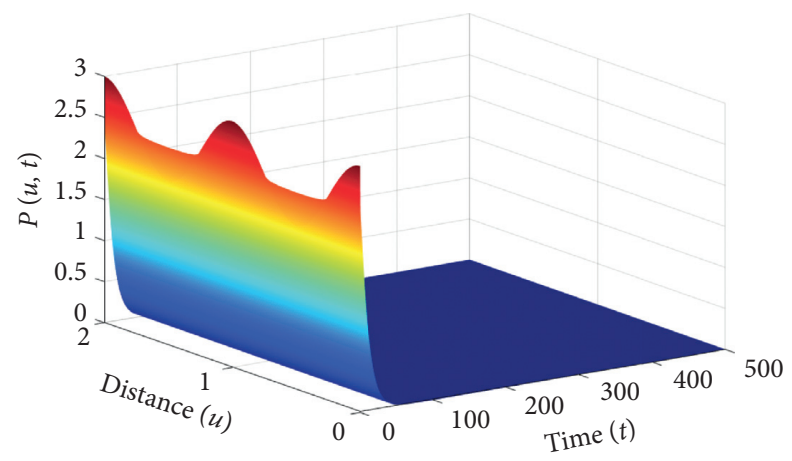

(e)

FIgURE 1: Taking Strategy $1\left(\mathscr{R}_{0} \leq 1\right)$, the equilibrium $\Omega_{0}=(1000,0,0,0,0)$ is asymptotically stable. (a) Target cells. (b) DENV-infected cells. (c) DENV particles. (d) Heterologous antibodies. (e) Homologous antibodies.

$$
\frac{\partial K}{\partial \overrightarrow{\mathscr{B}}}=\frac{\partial L}{\partial \overrightarrow{\mathscr{B}}}=\frac{\partial M}{\partial \overrightarrow{\mathscr{B}}}=\frac{\partial N}{\partial \overrightarrow{\mathscr{B}}}=\frac{\partial P}{\partial \overrightarrow{\mathscr{B}}}=0, \quad t>0, u=0,2 .
$$

We are using the following values of the parameters: $\delta=10, \xi=0.01, \varrho=0.3, \tau=5, \eta=3, \omega_{1}=0.3, \omega_{2}=0.1$, $\alpha_{1}=0.1$, and $\alpha_{2}=0.1$. The parameters $\mu, \lambda_{1}$, and $\lambda_{2}$ will be selected according to the following strategies:

Strategy 1. (Stability of $\Omega_{0}$ ) $\mu=0.00004, \lambda_{1}=0.0005$, and $\lambda_{2}=0.001$. For this set of parameters, we have $\mathscr{R}_{0}=0.2778<1, \quad \mathscr{R}_{1}=0.1389<1$, and $\mathscr{R}_{2}=0.1852<1$. Figure 1 shows that the solution of systems (2)-(6) converges to the equilibrium $\Omega_{0}=(1000,0,0,0,0)$. This shows that $\Omega_{0}$ is GAS according to Theorem 3. In this case, the DENV will be cleared.
Strategy 2. (Stability of $\Omega_{1}$ ) $\mu=0.0004, \lambda_{1}=0.0005$, and $\lambda_{2}=0.001$. With such choice, we get $\mathscr{R}_{0}=2.2222>1$, $\mathscr{R}_{1}=0.2469<1$, and $\mathscr{R}_{2}=0.4444<1$. Theorem 4 implies that $\Omega_{1}=(450,18.333,30.556,0,0)$ is GAS which is displayed in Figure 2. This will lead to the situation of persistent DENV infection but with an ineffective antibody immune response.

Strategy 3. (Stability of $\Omega_{2}$ ) $\mu=0.0004, \lambda_{1}=0.005$, and $\lambda_{2}=0.001$. Then, we compute $\mathscr{R}_{0}=2.2222>1$, $\mathscr{R}_{1}=1.2346>1$, and $\mathscr{R}_{2}=0.4444<\mathscr{R}_{1}$. Numerical results show that $\Omega_{2}=(555.556,14.815,20,2.346,0)$ exists. Moreover, Figure 3 shows that $\Omega_{2}$ is GAS which supports Theorem 5. Therefore, a persistent DENV infection with only effective heterologous antibody immune response is reached. 


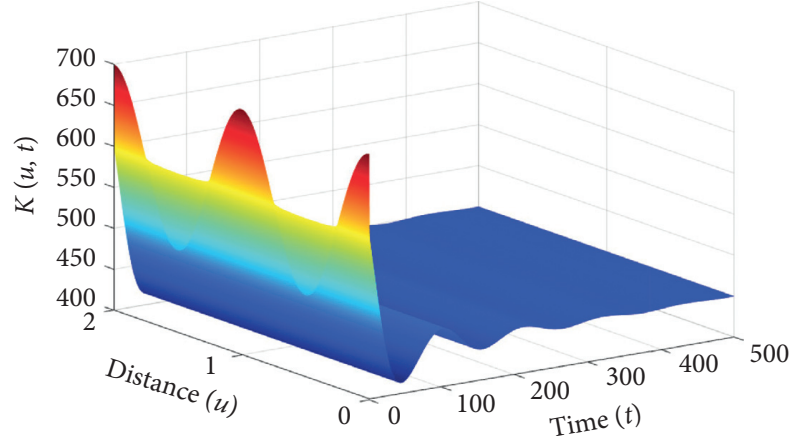

(a)

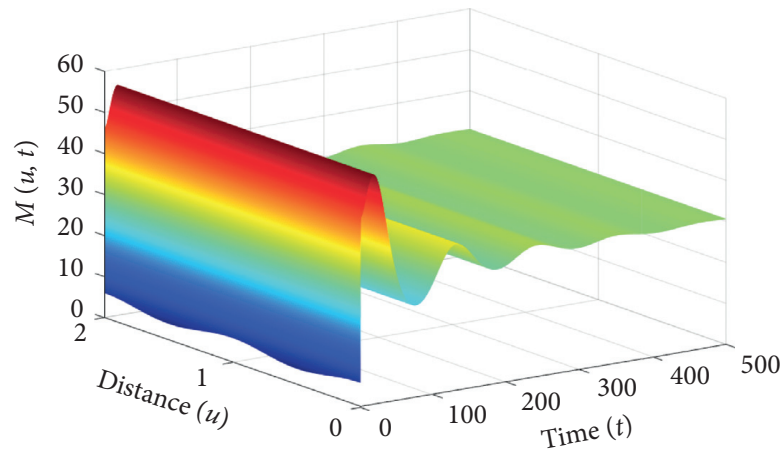

(c)

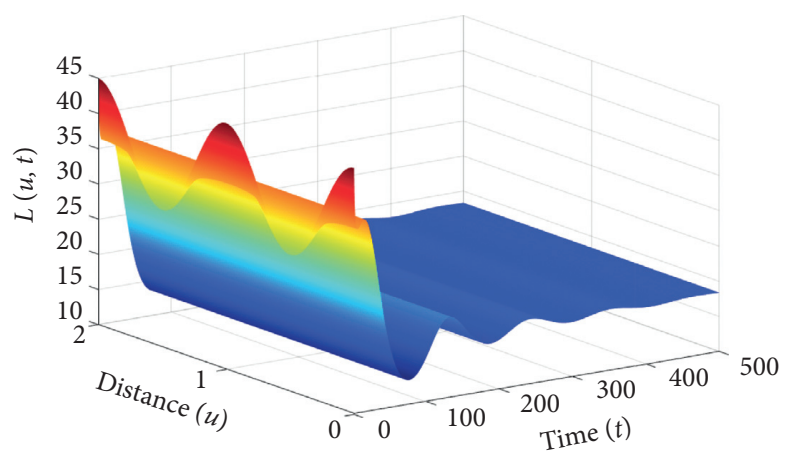

(b)

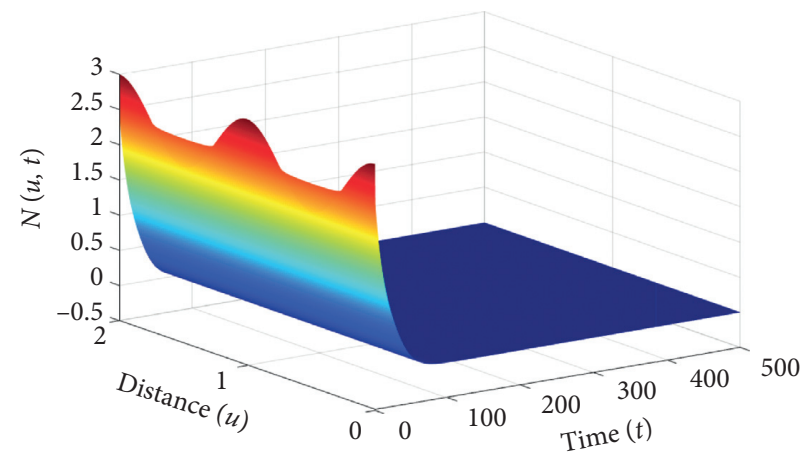

(d)

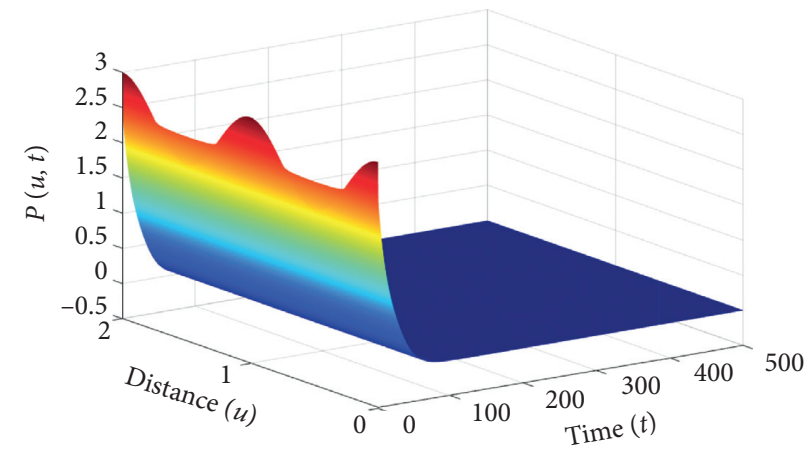

(e)

Figure 2: Taking Strategy $1\left(\mathscr{R}_{1} \leq 1<\mathscr{R}_{0}\right.$ and $\left.\mathscr{R}_{2} \leq 1\right)$, the equilibrium $\Omega_{1}=(450,18.333,30.556,0,0)$ is asymptotically stable. (a) Target cells. (b) DENV-infected cells. (c) DENV particles. (d) Heterologous antibodies. (e) Homologous antibodies.

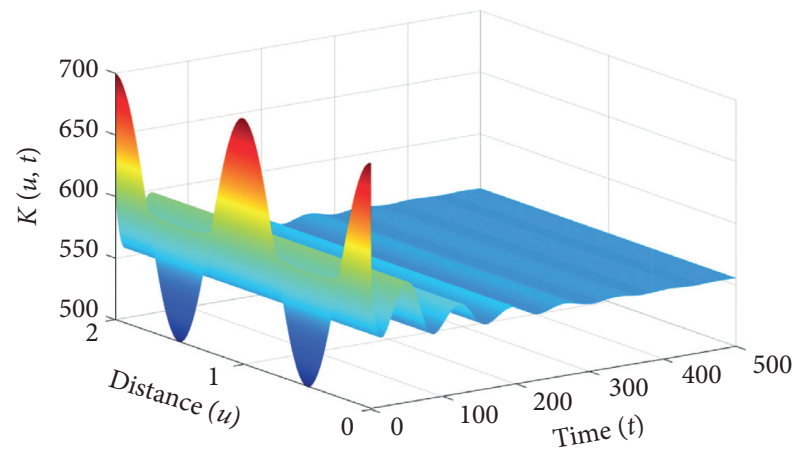

(a)

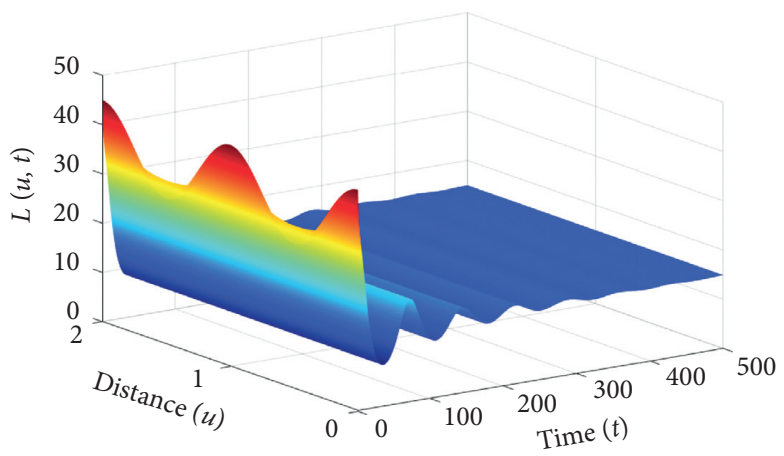

(b)

Figure 3: Continued. 


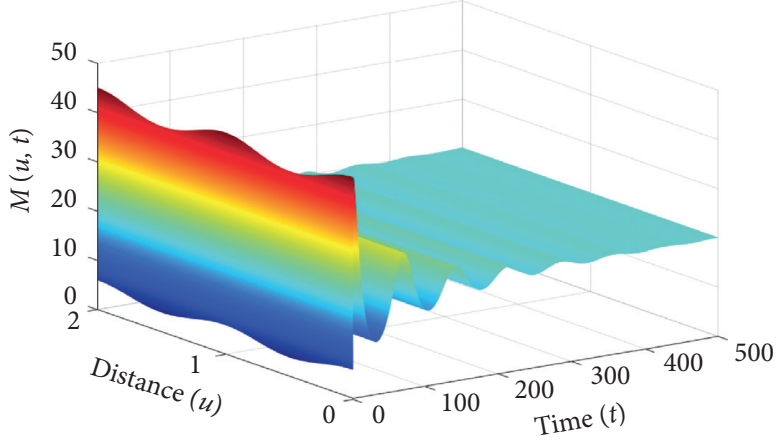

(c)

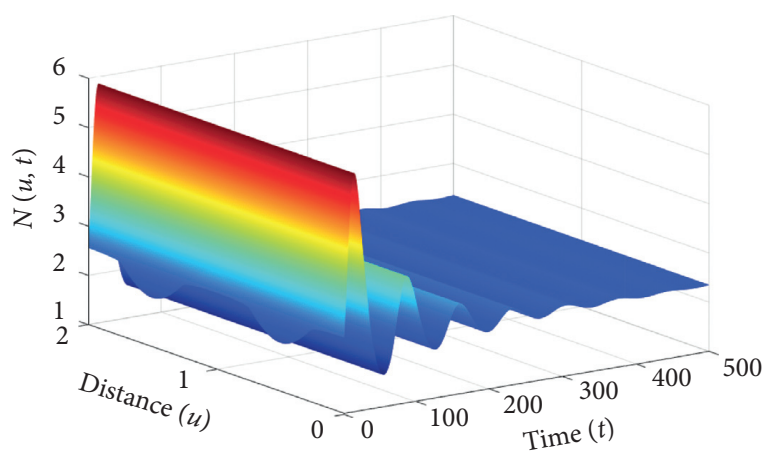

(d)

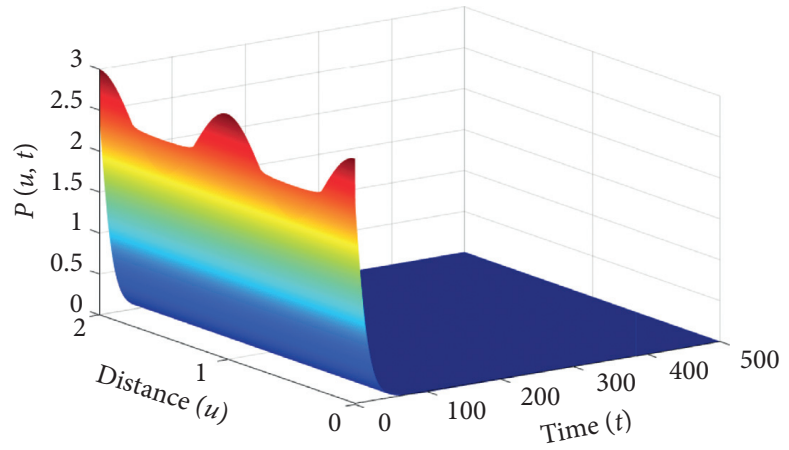

(e)

FIgURE 3: Taking Strategy $1\left(\mathscr{R}_{1}>1\right.$ and $\left.\mathscr{R}_{2} \leq \mathscr{R}_{1}\right)$, the equilibrium $\Omega_{2}=(555.556,14.815,20,2.346,0)$ is asymptotically stable. (a) Target cells. (b) DENV-infected cells. (c) DENV particles. (d) Heterologous antibodies. (e) Homologous antibodies.

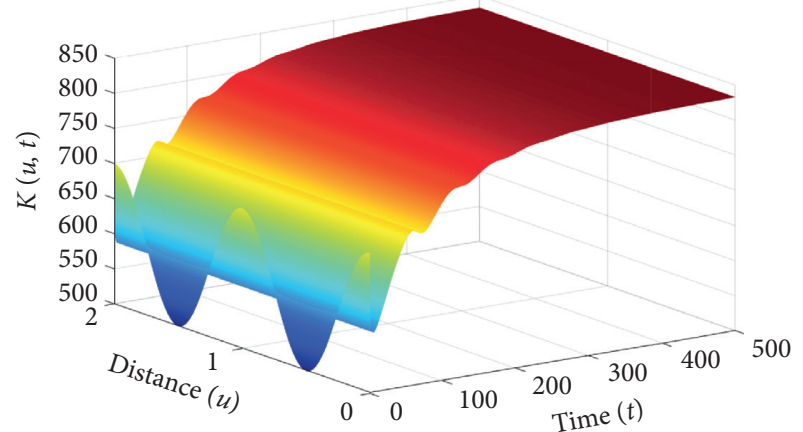

(a)

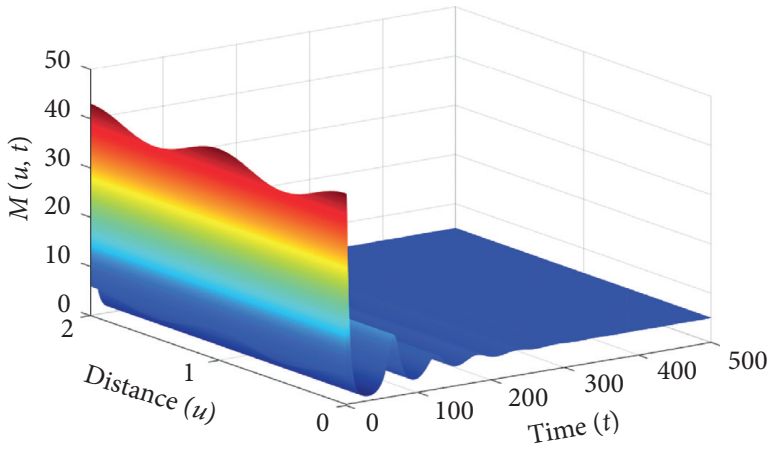

(c)

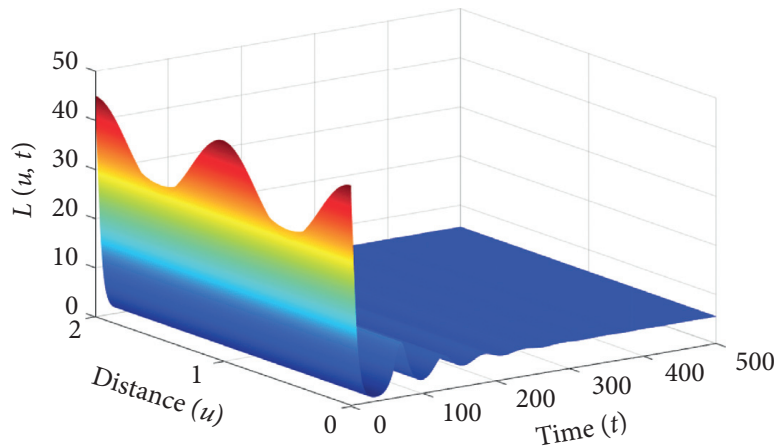

(b)

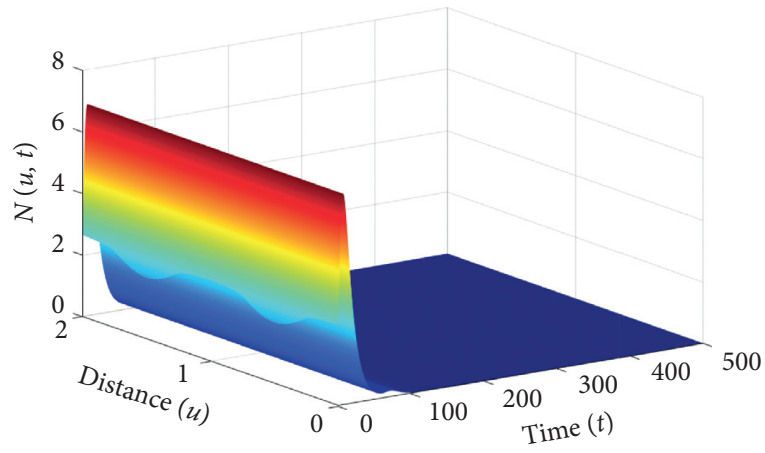

(d)

FIgUre 4: Continued. 


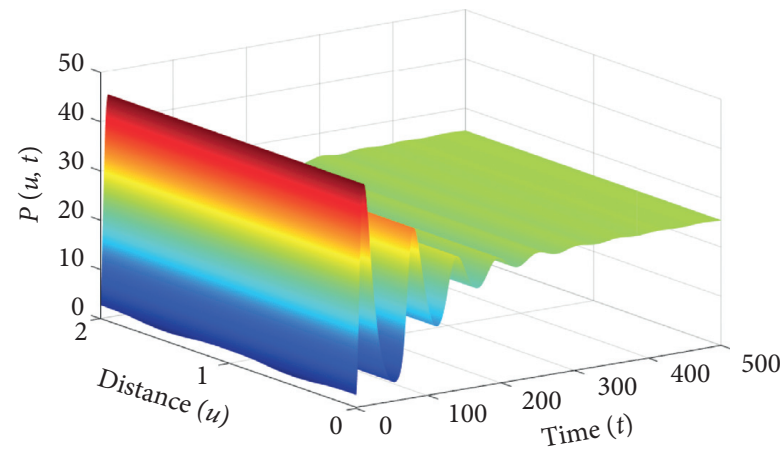

(e)

Figure 4: Taking Strategy $1\left(\mathscr{R}_{2}>1\right.$ and $\left.\mathscr{R}_{1} \leq \mathscr{R}_{2}\right)$, the equilibrium $\Omega_{3}=(833.333,5.556,5,0,25.556)$ is asymptotically stable. (a) Target cells. (b) DENV-infected cells. (c) DENV particles. (d) Heterologous antibodies. (e) Homologous antibodies.

Strategy 4. (Stability of $\Omega_{3}$ ) $\mu=0.0004, \lambda_{1}=0.01$, and $\lambda_{2}=0.02$. Then, we compute $\mathscr{R}_{0}=2.2222>1$, $\mathscr{R}_{1}=1.5873>1$, and $\mathscr{R}_{2}=0.8519>\mathscr{R}_{1}$. According to these data, $\Omega_{3}$ exists with $\Omega_{3}=(833.333,5.556,5,0,25.556)$. In Figure 4 , we show that $\Omega_{3}$ is GAS which is consistent with Theorem 6. In this case, a persistent DENV single infection with only effective homologous antibody immune response is reached.

\section{Conclusion}

A dynamical model to capture the behavior of secondary DENV infection was studied. The model incorporated the spatial mobility of DENV particles and cells. The model was given by five PDEs to describe the interaction between five compartments, target cells, DENV-infected cells, DENV particles, heterologous antibodies, and homologous antibodies. We first established that the model is biologically relevant by showing that the key variables of the model are nonnegative and bounded. We found that the model has four equilibria $\Omega_{i}, i=1,2,3,4$, and their existence and global stability are governed by three threshold parameters, $\mathscr{R}_{i}$, $i=1,2,3$. We performed the global stability analysis of the four equilibria by constructing suitable Lyapunov functions. We conducted some numerical simulations and found that the numerical results are fully aligned with the theoretical results. We summarize the obtained results in the following:

(i) The infection-free equilibrium $\Omega_{0}$ always exists, and it is GAS if $\mathscr{R}_{0} \leq 1$. This case corresponds to the healthy state where the DENV particles are cleared.

(ii) The persistent DENV infection equilibrium with ineffective antibodies $\Omega_{1}$ exists if $\mathscr{R}_{0}>1$, and it is GAS if $\mathscr{R}_{1} \leq 1$ and $\mathscr{R}_{2} \leq 1$. At this point, the DENV infection exists while the immune response is not active.

(iii) The persistent DENV infection equilibrium with only effective heterologous antibody $\Omega_{2}$ exists if $\mathscr{R}_{1}>1$, and it is GAS if $\mathscr{R}_{2} \leq \mathscr{R}_{1}$. This case leads to the situation where the DENV infection is chronic, while only heterologous antibody immune response is working.

(iv) The persistent DENV infection equilibrium with only effective homologous antibody $\Omega_{3}$ exists if $\mathscr{R}_{2}>1$, and it is GAS if $\mathscr{R}_{1} \leq \mathscr{R}_{2}$. At this point, the DENV infection is chronic with only an active homologous antibody immune response.

Our model can be extended to take into account the time delays during the DENV infection as

$$
\begin{aligned}
\frac{\partial K(u, t)}{\partial t}= & d_{K} \Delta K(u, t)+\delta-\mu K(u, t) M(u, t)-\xi K(u, t), \\
\frac{\partial L(u, t)}{\partial t}= & d_{L} \Delta L(u, t)+\mu e^{-k_{1} \psi_{1}} K\left(u, t-\psi_{1}\right) M\left(u, t-\psi_{1}\right)-\varrho L(u, t), \\
\frac{\partial M(u, t)}{\partial t}= & d_{M} \Delta M(u, t)+\tau e^{-k_{2} \psi_{2}} L\left(u, t-\psi_{2}\right)-\eta M(u, t)-\varpi_{1} M(u, t) \\
& \cdot N(u, t)-\varpi_{2} M(u, t) P(u, t) \\
\frac{\partial N(u, t)}{\partial t}= & d_{N} \Delta N(u, t)+\lambda_{1} M(u, t) N(u, t)-\alpha_{1} N(u, t) \\
\frac{\partial P(u, t)}{\partial t}= & d_{P} \Delta P(u, t)+\lambda_{2} M(u, t) P(u, t)-\alpha_{2} P(u, t)
\end{aligned}
$$


Here, it is assumed that a DENV contacts a target cell at time $t-\psi_{1}$, and the cell becomes infected at time $t$. Moreover, an infected cell at $t-\psi_{2}$ produces new infectious DENV particles at time $t$. The factors $e^{-k_{i} \psi_{i}}, i=1,2$, represent the survival rates of the cells during the delay periods.

\section{Data Availability}

Data sharing is not applicable to this article as no new data were created or analyzed in this study.

\section{Conflicts of Interest}

The authors declare that they have no conflicts of interest.

\section{Acknowledgments}

This study was funded by the Deanship of Scientific Research (DSR) at King Abdulaziz University, Jeddah (G: 274-1301441). The authors, therefore, acknowledge DSR for technical and financial support.

\section{References}

[1] World Health Organization, Dengue and Dengue Haemorrhagic Fever, WHO, Geneva, Switzerland, 2013, https://www. who.int/mediacentre/factsheets/fs117/en/.

[2] S. B. Halstead, "Dengue," The Lancet, vol. 370, no. 9599, pp. 1644-1652, 2007.

[3] S. K. Sasmal, Y. Takeuchi, and S. Nakaoka, "T-Cell mediated adaptive immunity and antibody-dependent enhancement in secondary dengue infection," Journal of Theoretical Biology, vol. 470 , pp. $50-63,2019$.

[4] S. Jindadamrongwech, C. Thepparit, and D. R. Smith, "Identification of GRP 78 (BiP) as a liver cell expressed receptor element for dengue virus serotype 2," Archives of Virology, vol. 149, no. 5, pp. 915-927, 2004.

[5] S. C. Kliks, W. E. Brandt, L. Wahl, A. Nisalak, and D. S. Burke, "Antibody-dependent enhancement of dengue virus growth in human monocytes as a risk factor for dengue hemorrhagic fever," The American Journal of Tropical Medicine and Hygiene, vol. 40, no. 4, pp. 444-451, 1989.

[6] J. M. Willey, L. M. Sherwood, and C. J. Woolverton, Microbiology, McGraw-Hill, New York, NY, USA, 7th edition, 2008.

[7] E. Navarro-Sánchez, P. Cedillo-Barrón, and L. Cedillo-Barreon, "Innate immune responses to dengue virus," Archives of Medical Research, vol. 36, no. 5, pp. 425-435, 2005.

[8] R. V. Gibbons and D. W. Vaughn, "Dengue: an escalating problem,” BMJ, vol. 324, no. 7353, pp. 1563-1566, 2002.

[9] N. Nuraini, H. Tasman, E. Soewono, and K. A. Sidarto, "A with-in host dengue infection model with immune response," Mathematical and Computer Modelling, vol. 49, no. 5-6, pp. 1148-1155, 2009.

[10] H. Ansari and M. Hesaaraki, "A with-in host dengue infection model with immune response and beddington-DeAngelis incidence rate," Applied Mathematics, vol. 3, no. 2, pp. 177-184, 2012.

[11] A. Mishra and S. Gakkhar, "A micro-epidemic model for primary dengue infection," Communications in Nonlinear Science and Numerical Simulation, vol. 47, pp. 426-437, 2017.
[12] B. R. Murphy and S. S. Whitehead, "Immune response to dengue virus and prospects for a vaccine," Annual Review of Immunology, vol. 29, no. 1, pp. 587-619, 2011.

[13] S. K. Sasmal, Y. Dong, and Y. Takeuchi, "Mathematical modeling on t-cell mediated adaptive immunity in primary dengue infections," Journal of Theoretical Biology, vol. 429, pp. 229-240, 2017.

[14] S. D. Perera and S. S. N. Perera, "Simulation model for dynamics of dengue with innate and humoral immune responses," Computational and Mathematical Methods in Medicine, vol. 2018, Article ID 8798057, 2018.

[15] S. Perera and S. S. N. Perera, "Mathematical modeling and analysis of innate and humoral immune responses to dengue infections," International Journal of Biomathematics, vol. 12, no. 7, Article ID 1950077, 2019.

[16] J. J. Thibodeaux, D. Nuñez, and A. Rivera, “A generalized within-host model of dengue infection with a non-constant monocyte production rate," Journal of Biological Dynamics, vol. 14, no. 1, pp. 143-161, 2020.

[17] H. E. Clapham, V. Tricou, N. Van Vinh Chau, C. P. Simmons, and N. M. Ferguson, "Within-host viral dynamics of dengue serotype 1 infection," Journal of the Royal Society Interface, vol. 11, Article ID 20140094, 2014.

[18] M. A. Nowak and C. R. M. Bangham, "Population dynamics of immune responses to persistent viruses," Science, vol. 272, no. 5258, pp. 74-79, 1996.

[19] World Health Organization, Dengue and Dengue Hemorrhagic Fever. Fact Sheet No. 117, WHO, Geneva, Switzerland, 2017, https://www.who.int/meidacentre/factsheets/fs117/en.

[20] T. P. Gujarati and G. Ambika, "Virus antibody dynamics in primary and secondary dengue infections," Journal of Mathematical Biology, vol. 69, no. 6-7, pp. 1773-1800, 2014.

[21] R. Ben-Shachar and K. Koelle, "Minimal within-host dengue models highlight the specific roles of the immune response in primary and secondary dengue infections," Journal of the Royal Society Interface, vol. 12, Article ID 20140886, 2015.

[22] R. Nikin-Beers and S. M. Ciupe, "Modelling original antigenic sin in dengue viral infection," Mathematical Medicine and Biology, vol. 35, no. 2, pp. 257-272, 2018.

[23] A. Mishra, "A within-host model of dengue viral infection dynamics," in Applied Analysis in Biological and Physical Sciences, J. Cushing, M. Saleem, H. Srivastava, M. Khan, and M. Merajuddin, Eds., Springer, New Delhi, India, 2016.

[24] M. Ceron Comez and H. M. Yang, "A simple mathematical model to describe antibody-dependent enhancement in heterologous secondary infection in dengue," Mathematical Medicine and Biology, vol. 36, pp. 411-438, 2019.

[25] M. Borisov, G. Dimitriu, and P. Rashkov, "Modelling the host immune response to mature and immature dengue viruses," Bulletin of Mathematical Biology, vol. 81, no. 12, pp. 49514976, 2019.

[26] K. Wang and W. Wang, "Propagation of HBV with spatial dependence," Mathematical Biosciences, vol. 210, no. 1, pp. 78-95, 2007.

[27] W. Wang and W. Ma, "Block effect on HCV infection by HMGB1 released from virus-infected cells: an insight from mathematical modeling," Communications in Nonlinear Science and Numerical Simulation, vol. 59, pp. 488-514, 2018.

[28] K. Hattaf and N. Yousfi, "Global stability for reaction-diffusion equations in biology," Computers \& Mathematics with Applications, vol. 66, no. 8, pp. 1488-1497, 2013.

[29] Y. Gao and J. Wang, "Threshold dynamics of a delayed nonlocal reaction-diffusion HIV infection model with both cell-free and cell-to-cell transmissions," Journal of 
Mathematical Analysis and Applications, vol. 488, no. 1, Article ID 124047, 2020.

[30] N. Bellomo, K. J. Painter, Y. Tao, and M. Winkler, "Occurrence vs. Absence of taxis-driven instabilities in a may-nowak model for virus infection," SIAM Journal on Applied Mathematics, vol. 79, no. 5, pp. 1990-2010, 2019.

[31] X. Ren, Y. Tian, L. Liu, and X. Liu, "A reaction-diffusion within-host HIV model with cell-to-cell transmission," Journal of Mathematical Biology, vol. 76, no. 7, pp. 1831-1872, 2018.

[32] W. Wei Wang and W. Wanbiao Ma, "Global dynamics of a reaction and diffusion model for an HTLV-I infection with mitotic division of actively infected cells," Journal of Applied Analysis \& Computation, vol. 7, no. 3, pp. 899-930, 2017.

[33] K. Hattaf, "Spatiotemporal dynamics of a generalized viral infection model with distributed delays and CTL immune response," Computation, vol. 7, no. 2, p. 21, 2019.

[34] Z. Wang, Z. Guo, Z. Guo, and H. Peng, "Dynamical behavior of a new oncolytic virotherapy model based on gene variation," Discrete \& Continuous Dynamical Systems-Series S, vol. 10, no. 5, pp. 1079-1093, 2017.

[35] Z. Xu and Y. Xu, "Stability of a $\mathrm{CD}^{+}{ }^{+} \mathrm{T}$ cell viral infection model with diffusion," International Journal of Biomathematics, vol. 11, no. 5, Article ID 1850071, 2018.

[36] Y. Zhang and Z. Xu, "Dynamics of a diffusive HBV model with delayed Beddington-DeAngelis response," Nonlinear Analysis: Real World Applications, vol. 15, pp. 118-139, 2014.

[37] H. L. Smith, Monotone Dynamical Systems: An Introduction to the Theory of Competitive and Cooperative Systems, American Mathematical Society, Providence, RI, USA, 1995.

[38] M. H. Protter and H. F. Weinberger, Maximum Principles in Differential Equations, Prentic Hall, Englewood Cliffs, NJ, USA, 1967.

[39] D. Henry, Geometric Theory of Semilinear Parabolic Equations, Springer-Verlag, New York, NY, USA, 1993.

[40] A. Elaiw and A. Al Agha, "Global analysis of a reactiondiffusion within-host malaria infection model with adaptive immune response," Mathematics, vol. 8, no. 4, p. 563, 2020.

[41] A. Korobeinikov, "Global properties of basic virus dynamics models," Bulletin of Mathematical Biology, vol. 66, no. 4, pp. 879-883, 2004.

[42] A. M. Elaiw and S. F. Alshehaiween, "Global stability of delaydistributed viral infection model with two modes of viral transmission and B-cell impairment," Mathematical Methods in the Applied Sciences, vol. 43, no. 11, pp. 6677-6701, 2020.

[43] A. M. Elaiw and N. H. AlShamrani, "Stability of a general CTL-mediated immunity HIV infection model with silent infected cell-to-cell spread," Advances in Difference Equations, vol. 2020, Article ID 355, 2020.

[44] A. M. Elaiw and N. H. AlShamrani, "Global stability of a delayed adaptive immunity viral infection with two routes of infection and multi-stages of infected cells," Communications in Nonlinear Science and Numerical Simulation, vol. 86, Article ID 105259, 2020.

[45] E. A. Barbashin, Introduction to the Theory of Stability, Wolters-Noordhoff, Groningen, The Netherland, 1970.

[46] J. P. LaSalle, The Stability of Dynamical Systems, SIAM, Philadelphia, PA, USA, 1976.

[47] A. M. Lyapunov, The General Problem of the Stability of Motion, Taylor \& Francis, Ltd., London, UK, 1992.

[48] J. Liang, J. Wei, and J. Wei, "Lyapunov functional for virus infection model with diffusion and state-dependent delays," Mathematical Biosciences and Engineering, vol. 16, no. 2, pp. 947-966, 2019.
[49] A. M. Elaiw and A. D. Al Agha, "Analysis of a delayed and diffusive oncolytic M1 virotherapy model with immune response," Nonlinear Analysis: Real World Applications, vol. 55, Article ID 103116, 2020.

[50] A. M. Elaiw, A. D. Hobiny, and A. D. Al Agha, "Global dynamics of reaction-diffusion oncolytic M1 virotherapy with immune response," Applied Mathematics and Computation, vol. 367, Article ID 124758, 2020. 